\title{
THE IMPACT OF ECB
}

\section{MONETARY POLICY}

\section{DECISIONS AND}

\section{COMMUNICATION ON}

THE YIELD CURVE

by Claus Brand,

Daniel Buncic

and Jarkko Turunen 
E UROPEANCENTRAL BANK

\section{WORKING PAPER SERIES}

NO 657 I JULY 2006

\section{THE IMPACT OF ECB \\ MONETARY POLICY \\ DECISIONS AND \\ COMMUNICATION ON THE YIELD CURVE'}

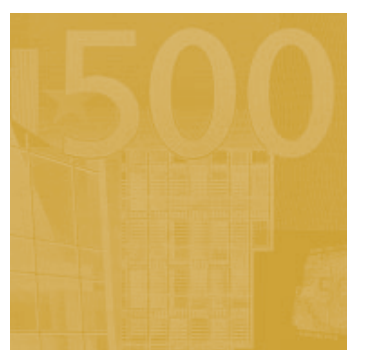

by Claus Brand',

Daniel Buncic ${ }^{3}$

and Jarkko Turunen ${ }^{4}$

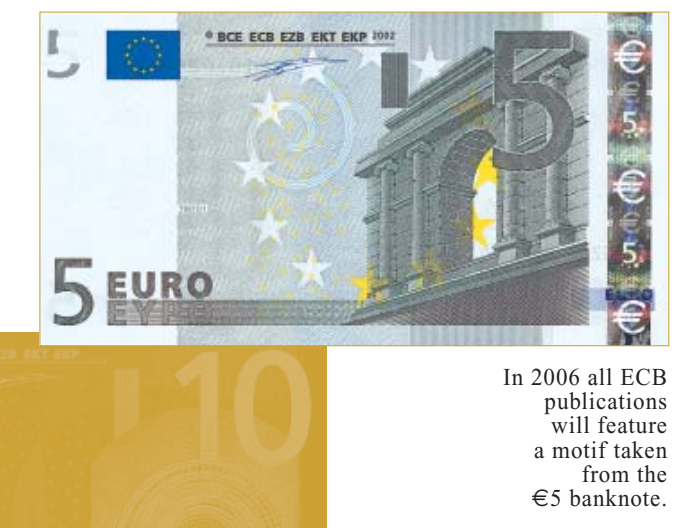

This paper can be downloaded without charge from http://www.ecb.int or from the Social Science Research Network electronic library at http://ssrn.com/abstract_id $=913330$

I This paper reflects the views of the authors and does not necessarily reflect the views of the European Central Bank. We are grateful to Vincent Brousseau, ECB, for providing us with the intraday data used throughout this study. We would like to thank an anonymous referee of the ECB Working Paper Series, Magnus Andersson, Michael Ehrmann, Juan-Angel Garcia, Stefan Gerlach, Refet Gürkaynak, Philippe Moutot, Diego Rodríguez Palenzuela, Huw Pill, Eric Swanson, Thomas Werner, and ECB seminar participants for helpful comments.

2 Corresponding author: European Central Bank, Kaiserstrasse 29,603।I Frankfurt am Main, Germany; e-mail: claus.brand@ecb.int 3 School of Economics, University of New South Wales, Sydney Australia; e-mail: d.buncic@unsw.edu.au 4 European Central Bank; Kaiserstrasse 29, 603 II Frankfurt am Main, Germany; e-mail: jarkko.turunen@ecb.int 
(C) European Central Bank, 2006

\section{Address}

Kaiserstrasse 29

60311 Frankfurt am Main, Germany

\section{Postal address}

Postfach 160319

60066 Frankfurt am Main, Germany

\section{Telephone}

+496913440

\section{Internet}

http://www.ecb.int

\section{Fax}

+496913446000

\section{Telex}

$411144 \mathrm{ecb} d$

All rights reserved.

Any reproduction, publication and reprint in the form of a different publication, whether printed or produced electronically, in whole or in part, is permitted only with the explicit written authorisation of the ECB or the author(s).

The views expressed in this paper do not necessarily reflect those of the European Central Bank.

The statement of purpose for the ECB Working Paper Series is available from the ECB website, http://www.ecb.int.

ISSN 1561-0810 (print)

ISSN 1725-2806 (online) 


\section{CONTENTS}

Abstract 4

Non-technical summary 5

1 Introduction $\quad 7$

2 Some institutional features of ECB monetary policy announcements and communication $\| 0$

3 Extracting news from the money market yield curve

3.1 Principal components

3.2 Recursive regressions

3.3 Separate decision and communication windows

3.4 Jobless claims

4 Evolution of monetary policy news over time

4.1 Main results

4.2 Interpreting specific events

5 Impact of monetary policy news on longer-term yields

6 Conclusions

References

Appendix

Figures and tables

European Central Bank Working Paper Series 


\begin{abstract}
We analyse high-frequency changes in the euro area money market yield curve on dates when the ECB regularly sets and communicates decisions on policy interest rates to construct different indicators of monetary policy news relating to policy decisions and to central bank communication. The indicators show that ECB communication during the press conference may result in significant changes in market expectations of the path of monetary policy. Furthermore, our results suggest that these changes have a significant and sizeable impact on medium to long-term interest rates.
\end{abstract}

Keywords: money market rates, yield curve, ECB, central bank communication. JEL Classification: E43, E58. 


\section{Non-technical summary}

To underpin their commitment to low and stable inflation, central banks have sought to improve the transparency of their monetary policy strategies through - among other important elements - appropriate communication with financial markets and the public. Central bank communication has thus become a key element in contributing to stabilise expectations as to how the central bank responds to changes in the state of the economy.

We construct multi-dimensional indicators of monetary policy news which capture information relating to monetary policy decisions and communication at different maturity horizons. In particular, we analyse high-frequency changes in money market rates during days when the Governing Council of the European Central Bank (ECB) decides on policy interest rates and - in the context of its regular press conference taking place shortly afterwards - explains this decision to the public. The indicators that we construct relate not only to the extent to which current policy decisions have been anticipated, but also to the extent to which expectations of the path of monetary policy have been revised as a result of communication that accompanies the decision. Having constructed several multidimensional indicators of monetary policy news we use them to analyse the impact of policy action and communication on expectations of the level of interest rates at various time horizons. Measuring the impact of monetary policy news on the nominal yield curve is useful in assessing the success of a central bank in managing expectations about future policy rates and in analysing the financial market participant's understanding of the central bank's intentions.

Our study adds to the empirical literature on central bank communication in two important ways: First, the use of intraday data together with the institutional feature that the ECB announces and explains policy decisions on two different points in time during the day allows us to directly construct measures of policy news that relate separately to the decision and communication dimensions. This direct measure can be compared with measures based on econometric analysis of changes of the money market yield curve proposed in the literature. For the US, these studies show that intraday changes in federal fund futures on FOMC meeting days can be characterised by two or more factors, with one factor relating to the Federal Reserve's policy decisions and the other to communication about the future path of policy interest rates.

Second, our study importantly complements existing evidence about the financial market impact of the ECB's monetary policy. We provide first evidence of news about ECB monetary policy using multidimensional indicators based on money market data. This approach has two key advantages compared with existing literature. By explicitly focussing on the multidimensionality of monetary policy news we provide a richer description of news than existing studies of ECB monetary policy that focus on single-factor indicators constructed from (daily) changes in short term money market rates or surveys of financial market participants. Furthermore, by using market interest rates we circumvent the inherent subjectivity of indicators based on a context based analysis of ECB statements used to capture the tone in ECB communication. The meaning of such subjective indicators may not coincide with how financial markets understand new information about monetary policy.

We arrive at a number of important conclusions. First, the size and evolution of the indicators of monetary policy news developed in this paper suggests that market participants have recently been in a better position to anticipate the course of ECB monetary policy than in the 
past. They also confirm the view that ECB communication during the press conference may result in significant changes in market expectations of the path of monetary policy. Second, our results support the use of these indirect econometric methods in constructing measures of news from central bank communication. Indeed indicators of news related to ECB communication derived using different econometric methodologies give information that is very similar to the information from the direct approach. Third, as regards the impact of monetary policy news on the nominal yield curve, our results show that changes in expectations of the course of monetary policy triggered by the ECB's communication have a significant and sizeable impact on medium to long-term interest rates. This impact is similar in size to that measured for the US. At the same time, news about immediate policy decisions have an impact only at shorter maturities. Overall, these results suggest that ECB actions and communication have been consistent, leading financial market participants to closely follow and to react to regular ECB communication in forming their expectations of future policy interest rates. 


\section{Introduction}

When the Governing Council of the European Central Bank (ECB) met to discuss monetary policy on 6 April 2006, financial market participants did not expect any changes in key ECB interest rates. As a result, following the announcement not to change interest rates, there was no change in money market interest rates. However, prior to the April meeting, expectations of an interest rate hike at the May 2006 Governing Council meeting had emerged. Following the President's statement during the monthly press conference (starting later on 6 April) that "the current suggestions regarding the high probability of an increase of rates in our next meeting do not correspond to the present sentiment of the Governing Council", interest rates throughout the entire money market maturity spectrum dropped significantly, reflecting a downward adjustment in expectations of short-term interest rates. Quite remarkably, these changes were not triggered by actual policy decisions, but rather by how the stance of monetary policy was communicated.

This example shows that in constructing indicators of monetary policy news it is crucial to capture all information used by financial market participants to form expectations about future policy. It seems particularly important to capture monetary policy news stemming not only from decisions, but also from central bank communication. Central bank communication has become a key element in contributing to stabilise private sector expectations as to how the central bank responds to changes in the state of the economy. This element is crucial from a policy perspective, since the success of monetary policy does not depend on the effective control of the overnight rate, but rather on shaping market expectations of how interest rates and inflation are likely to evolve over time. Indeed, Woodford (2003) considers central banking to be exclusively about the "management of expectations" : "Not only do expectations about policy matter, but ... very little else matters".

We use intraday changes in money market rates to construct indicators of news about monetary policy stemming from the policy decisions and official communication of the ECB and study their impact on the yield curve. Given the enhanced role of communication and private sector expectations in the practise of central banking, the indicators that we construct relate not only to the extent to which current policy decisions have been anticipated, but also to the extent to which expectations of the path of monetary policy have been revised as a result of communication that accompanies the decision. This separation turns out to be very important for understanding the nature of new information about ECB monetary policy.

The main value added of our paper is two-fold. First, the institutional feature that the ECB announces and explains policy decisions on two different points in time during the day allows us to directly construct measures of policy news (based on intraday changes in the money market yield curve) that relate separately to the decision and to the communication dimension. This allows us to compare this direct measure with indirect measures that have been constructed using econometric methods that comprise both decision and communication events, as suggested by Gürkaynak et al. (2005) and Gürkaynak (2005). For the US, these studies show that intraday changes in federal fund futures on FOMC meeting days can be characterised by two or more factors - with one factor relating to the Federal Reserve's policy decisions, the other to communication about the future path of policy interest rates.

Second, this study complements existing evidence of the financial market impact of the ECB's monetary policy decisions and communication. We provide first evidence of news about 
ECB monetary policy using multidimensional indicators based on intraday money market data. Our approach has two main advantages with regards to the existing literature. By explicitly focussing on the multi-dimensionality of monetary policy news, relating separately to different policy horizons, we provide a richer description of news about monetary policy than existing studies of ECB monetary policy, focussing on single-factor indicators. A number of studies have used single-factor indicators to study very short-term news about policy decisions. These indicators are commonly based on information derived from (daily) changes in money market interest rates or surveys of financial market participants. For example, Perez-Quiros and Sicilia (2002) use daily changes in the 1-month EONIA swap rates and Ehrmann and Fratzscher (2003) the difference between mean expectations of the policy decision among ECB watchers from the Reuters poll one week before the decision and the actual policy outcome. Other studies have used information from EURIBOR futures (see Bernoth and von Hagen, 2004; Wilhelmsen and Zaghini, 2005)

By using market interest rates we also circumvent the inherent shortcomings of subjective indicators of the tone in ECB communication that have been constructed using a context based analysis of ECB statements. Several authors have constructed such subjective indicators based on counting code words in the ECB introductory statement or the Monthly Bulletin editorial ((see Heinemann and Ullrich, 2005), Rosa and Verga (2005) Gies (2005); Meyer (2004) and Gerlach (2004)). Using the indicator developed in Gies (2005), both Gies (2005) and Sebestyén and Sicilia (2005) find that the tone of ECB communication has an impact on both short and longterm interest rates in the euro area. Alternatively, Ehrmann and Fratzscher (2005) and Ehrmann and Fratzscher (2006) construct indicators of the tone in speeches and statements made by Governing Council members in the period between Governing Council meetings. They use these indicators to construct measures of dispersion about the views of the economic outlook and monetary policy and study the implications for the predictability of monetary policy. However, there are shortcomings to such approaches. The meaning of the subjective indicators may not coincide with how financial markets understand new information about monetary policy. By construction, subjective indicators cannot possibly reflect all the information that is used by financial markets when forming expectations about monetary policy. First, in choosing a scale for the subjective indicator, these indicators rely on the assumption that a central bank uses language and certain code words in a highly consistent manner. Second, these indicators confound information as to whether the tone detected in announcements is meant to explain a decision which has been taken or to provide signals about an upcoming decision in the next meeting or policy actions at much longer horizons.

The use of high-frequency money market data for the decomposition allows us to identify the news component of ECB communication without relying on subjective indicators. In fact, we treat changes in money market yields around the time of the release of the Governing Council's interest rate decision and the press conference as the information capturing monetary policy. This approach can be justified by the absence of other relevant data releases during this narrow time window.

In constructing indicators of monetary policy news relating to various policy horizons, we decompose intraday changes in the euro area money market yield curve on ECB Governing Council meeting days into (up to) three dimensions: news related to the level of the ECB policy interest rate, which we will call "jump" news, news related to the "timing" of policy decisions, and finally news related to the "path" reflecting monetary policy signals pertaining to 
longer horizons. This decomposition is done using two separate methodologies: first, using the principal components based method in Gürkaynak et al. (2005) and second, using a recursive regression approach similar to Gürkaynak (2005). In addition, taking advantage of the salient feature that the ECB announces and explains policy decisions on two different time instances, we construct separate indicators of monetary policy for decision and communication effects on the basis of two different time windows.

Having constructed several multidimensional indicators of monetary policy news, we use them to analyse the impact of monetary policy decisions and communication on the yield curve. We focus on the impact of policy news on interest rates of different maturities. This aim differs from earlier studies of the euro area. Sebestyén and Sicilia (2005) and Andersson et al. (2006), for example, look at the impact on volatility. ${ }^{1}$ In contrast to these studies we focus mainly on the impact of policy news and statements on expectations of the level of interest rates at various time horizons. This focus on the level is more helpful for assessing how successful a central bank is in managing expectations and to analyse whether financial market participants understand the central bank's action and intentions.

Our results suggest that, according to the size and evolution of indicators of monetary policy news developed in this paper, market participants have recently been in a better position to anticipate the course of monetary policy than in the past. They also confirm the view that ECB communication during the press conference may result in significant changes in market expectations of the path of monetary policy. Furthermore, our results support the use of these indirect econometric methods in constructing measures of news from central bank communication. Indeed indicators of news related with ECB communication derived using different econometric methodologies give information that is very similar to the information from the direct approach. Finally, as regards the impact of monetary policy news on the nominal yield curve, our results show that changes in expectations of the course of monetary policy triggered by the ECB's communication have a significant and sizeable impact on medium to long-term interest rates. This impact is similar in size to that measured for the US. At the same time, news about immediate policy decisions have an impact only at shorter maturities.

The remainder of the paper is structured as follows. In section 2 we motivate different ways of constructing indicators of monetary policy news relating to different time horizons. To this end, we review in some more detail, how and when the ECB announces and explains its monetary policy decisions taken in its regular monthly rate-setting meeting and how money market rates tend to evolve on those days. In section 3 we describe the various methods adopted to construct the monetary policy indicators. In section 4 we discuss how the resulting indicators have evolved over time, compare and validate them by explaining specific events. Based on these indicators, in section 5 we provide an analysis of the impact of monetary policy news on the euro area yield curve. We conclude in section 6 . We present a number of technical details in the Appendix.

\footnotetext{
${ }^{1}$ Sebestyén and Sicilia (2005) use daily data and an EGARCH model of volatility of a number of asset prices with different maturities and find that ECB communication result in increased market volatility. Similarly, Andersson et al. (2006), using intraday data, find that volatility in German bond markets increases within tight time windows around the ECB press conference.
} 


\section{Some institutional features of ECB monetary policy announce- ments and communication}

We analyse intraday changes in the money market yield curve on days, when the Governing Council, the decision-making body of the ECB, meets to discuss monetary policy. The meetings now take place once a month. The timing of specific events on those days is crucial for the construction of indicators of monetary policy news. Immediately following the meeting, the ECB announces and explains its decisions on two different time instances during the same day (see Figure 1). The ECB publishes its monetary policy decision at 13.45. The publication consists of a short press release that simply states the interest rate decision. Three quarters of an hour later, beginning at 14:30, the ECB holds a press conference where the President of the ECB explains this decision in detail. At the beginning of the press conference the President of the ECB reads an introductory statement that provides a comprehensive summary of the assessment of economic and monetary developments and explains the monetary policy decision taken by the Governing Council. This part of the press conference is usually over by about 14:45. Following the introductory statement, the President answers journalists' questions which relate mainly to considerations underlying the ECB's policy decision.

The use of intraday data allows us to separate the impact of the publication of ECB decisions and communication. Based on the institutional features of ECB communication we use different time windows to construct measures of monetary policy news (see Figure 1). First, we use a wide time window from 13:35-15:50 and the statistical techniques proposed by Gürkaynak et al. (2005) and Gürkaynak (2005) to decompose the information set on monetary policy. This time window is used only on Governing Council meeting days that comprise a press conference. When no press conference took place, the window ends at 14:05. ${ }^{2}$ Second, we consider two separate narrow time windows, which we label "decision window" (from 13:35-14:05) and "communication window" (from 14:20-15:50). This separation allows us to directly measure the effect of news about policy decisions and communication on financial assets without needing to resort to statistical tools.

To underpin the precise length of the respective windows, it is illustrative to look at how fast market rates react to news about ECB monetary policy. Figure 2 shows the minute to minute evolution of the 60 day rate on two specific instances (a description of the data is provided in section 3). The dashed line (RHS) in Figure 2 shows the intraday evolution on 10 May 2001 when the ECB decided to lower its key policy interest rates by 25 basis points. This decision had not been anticipated by financial market participants, which is clearly visible in the sharp drop in the 60 day rate at the time of the publication of the decision. The chart also shows that it took less than 10 minutes for money market interest rates to adjust to the announced change in key ECB interest rates and that our choice of the length of the decision window allows for a full adjustment of expectations to news during that time. At the same time, the window is narrow enough to ensure that changes in expectations only relate to news about monetary policy. We

\footnotetext{
${ }^{2}$ Before November 2001 policy setting meetings of the Governing Council took place at a fortnightly frequency, whereas press conference took place only once a month (after the first meeting of the month). After November 2001 - with the exception of the summer recess and around the turn of the year - the meetings and press conferences have regularly taken place on the first Thursday of the month. The time intervals between the observations are therefore not equal.
} 
can also see from Figure 2 that on 10 May 2001 money market rates did not change in response to the explanation of the interest rate decision given subsequently during the press conference starting at 14:30.

As an example of how money market rates have reacted to news during the ECB's press conference, rather than the publication of its decision about key interest rates, the solid line (LHS) in Figure 2 shows the intraday evolution of the 60 day rate on 6 April 2006. While financial markets had attached a significant probability of an interest rate hike in May 2006, no immediate change was expected at the meeting on 6 April. As there was, in fact, no change in interest rates on that day market interest rates remained stable at the time of the publication of the ECB decision at 13:45. The first ten minutes of the press conference, i.e. the time it took the President to read out the Introductory Statement (14:32-14:42 on that occasion), also did not result in major changes in money market interest rates. Following the start of the questions and answers session, however, when the President stated that "the current suggestions regarding the high probability of an increase of rates in our next meeting do not correspond to the present sentiment of the Governing Council", money market rates declined visibly. The 60 day rate dropped by about 8 basis points within the next 45 minutes and stabilised at the lower level roughly an hour after the press conference had started.

These episodes illustrate two important features. First, they show that the adjustment in market rates takes place immediately after news about monetary policy become available. This observation is in line with empirical results showing that the euro area money market is efficient in incorporating new information (for further evidence for different segments of the euro area money market see Bernoth and von Hagen (2004), Hartmann et al. (2001) and Sebestyén (2006). Second, it shows that the length of the time windows chosen is wide enough to capture the time the market needs to absorb news and, at the same time, narrow enough to prevent that policy news are contaminated by other news releases during the same day. The release of the weekly US jobless claims at 14:30 is the only exception. We provide an assessment of the impact of this specific data release in subsection 3.4 and section 5 .

\section{Extracting news from the money market yield curve}

We use three different methodologies to extract news from the money market yield curve. Let $f_{t}^{\tau}$ denote the implied 10 day money market forward rate $\tau$ days ahead as extracted from the discount curve. Furthermore, let $\Delta f_{t}^{\tau}$ denote the change in the implied forward rate over the time window under consideration. The frequently used changes in forward rates 10, 30 and 150 days ahead are thereby labelled $\Delta f_{t}^{10}, \Delta f_{t}^{30}$ and $\Delta f_{t}^{150}$.

The intraday interest rate data that are employed in this study consist of real time quotes (of deposit and swap rates) from Reuters, observed at five minute intervals. The data are unsmoothed, but have been filtered for mispriced quotes according to the algorithm developed by Brousseau (2002). The data was interpolated as in Brousseau (2006) to yield a discount curve with a maturity grid of 10 days, covering maturities from the overnight rate up to 10 years. ${ }^{3}$ As we focus exclusively on regular Governing Council meeting days, the policy decision taken

\footnotetext{
${ }^{3}$ We are grateful to Vincent Brousseau (ECB) for providing us with this dataset. We applied a mild smoothing spline to the discount curve.
} 
at the unscheduled Governing Council meeting on 17 September 2001 was excluded from the sample. Our sample period thus covers the regular Governing Council meeting days from 30 November 2000 through to 4 May 2006. We identify changes in the forward rates within the time windows with news about monetary policy. Overall, changes in forward rates may also comprise other influences, including changes in forward premia over the respective time windows. However, a decomposition of forward rates into such time-varying premia and a risk-free rate is beyond the scope of this paper.

\subsection{Principal components}

Gürkaynak et al. (2005) have recently proposed a method for extracting jump and path news based on principal components analysis of changes in the yield curve over certain time windows. The corresponding factor model can be expressed in the following form

$$
Y=F \Omega^{\prime}+\eta
$$

where $Y$ is a $T \times N$ matrix of data, $F$ is a $T \times k$ matrix of unobserved factors with $k<N$, $\Omega^{\prime}$ is a $k \times N$ matrix of factor loadings and $\eta$ is a $T \times N$ matrix of idiosyncratic disturbance terms. Matrix $\Omega$ contains the eigenvectors of $\operatorname{Cov}(Y)$ and $F$ is computed in the standard way as $F=Y \Omega$.The $Y$ matrix was chosen in line with the data matrix used by Gürkaynak et al. (2005) and consists of $\left\{\Delta f_{t}^{10}, \Delta f_{t}^{30}, \Delta f_{t}^{150}, \Delta f_{t}^{200}, \Delta f_{t}^{270}, \Delta f_{t}^{360}\right\}_{t=1}^{T}$. The corresponding changes in the forward rates are measured as changes over the wide time window, 13:35-15:50, covering the announcement and the press conference.

A priori, considering the results obtained from previous studies (see for example Gürkaynak et al., 2005)), we expect that two factors are sufficient to account for most of the variation in the $Y$ series. However, we also employed the reduced rank test of Cragg and Donald (1997), which is described in more detail in Gürkaynak et al. (2005, pp. 87-88). The test fails to reject the null hypothesis of two factors being sufficient to account for most of the variation in $Y$ at the 5\% level of significance. ${ }^{4}$

Since these estimated factors have no structural interpretation, Gürkaynak et al. (2005) suggest a factor rotation so that the first rotated factor corresponds to what we label 'jump' news and the second to 'path' news. We define $Z=F U$ where $U$ is a $2 \times 2$ orthogonal matrix, $F=\left(f_{1}, f_{2}\right)$ are the factors obtained from principal components analysis and $Z=\left(z_{1}, z_{2}\right)$ are the rotated factors. The interpretation and labels of the rotated factors is based on a choice of $U$ which ensures that the second structural factor $z_{2}$ does not load into the short-term interest rate. Details about the computation of this rotation are given in the Appendix. Note that, since $f_{1}$ and $f_{2}$ are orthogonal by construction, the rotated factors $z_{1}$ and $z_{2}$ will also be orthogonal.

\footnotetext{
${ }^{4}$ The details of the test are available upon request. We would like to thank Eric Swanson for providing the Matlab code to perform this test. We have given preference to this test rather then the more recently proposed tests by, for example, Bai and $\mathrm{Ng}$ (2002), as the column dimension of $Y$ is fairly small and the asymptotic distribution of these tests relies upon both $T$ and $N$ going to infinity.
} 


\subsection{Recursive regressions}

An alternative method of extracting monetary policy news of comparable nature is suggested in Gürkaynak (2005) based on a recursive regression scheme. To identify jump, timing and path news, we define $\Delta f_{t}^{30} \equiv j u m p_{t}$ and extract timing $g_{t}$ and $p a t h_{t}$ news as the residuals from the following OLS regressions:

$$
\begin{aligned}
\Delta f_{t}^{10} & =\alpha_{0}+\alpha_{1} j u m p_{t}+\text { timing }_{t} \\
\Delta f_{t}^{150} & =\gamma_{0}+\gamma_{1} j u m p_{t}+\gamma_{2} \text { timing }_{t}+\text { path }
\end{aligned}
$$

whereby, $\Delta f_{t}^{\tau}$ is again measured as changes in forward rates over the wide time window.

Hence, starting with the definition of the jump news, the timing component is obtained by filtering out the jump component of the changes in the 10-day rate. ${ }^{5}$ The extraction of timing news on the basis of the choice of maturities for this decomposition is mainly applicable to the period prior to November 2001, when the rate setting meeting took place at fortnightly frequencies. After that date the Governing Council has met once a month to discuss interest rates. Therefore, the non-zero timing news allowed during this time period should be treated with caution. ${ }^{6}$

As a third component, a path component is defined as the residual from regressing the 5-months rate on the jump and timing components. Thus a jump component would relate to unanticipated changes in the policy interest rate, timing to the changes that may have been anticipated in terms of their level, but not their timing. Finally, path relates to changes in money market interest rates of longer-term maturities not owing to jump and timing.

This decomposition is reflected in the recursive scheme in (2) and (3). The $\bar{R}^{2}$ of the regressions in (2) and (3) are 0.58 and 0.31 , respectively, indicating that $\Delta f_{t}^{10}$ is largely comprised of jump news and $\Delta f_{t}^{150}$ of path news. These regressions suggest that for the longer horizons it is important to clean out the impact of short term news of monetary policy. In particular, nearly a third of the changes in forward rates at the 150 day horizon are explained by changes at shorter horizons.

\subsection{Separate decision and communication windows}

The previously discussed methods of extracting jump and path news are purely statistical. However, because of the salient feature that the ECB decisions of policy interest rates are an-

\footnotetext{
${ }^{5}$ Financial markets may anticipate a policy move of a certain size by a specific date. Markets may, however, not fully anticipate the precise timing of this decision. This might lead to policy news in terms of the timing component, while the jump component would be uninformative. For example, an anticipated 50 basis points cut in policy interest rates may have been anticipated by a certain date, but whether it is delivered fully on the first meeting within a month, or on the second meeting within a month, or in two consecutive 25 basis point cuts might not be fully anticipated.

${ }^{6}$ In section 4 we show that measured timing news are mostly of an insignificant size after November 2001. There is only one major exception related to the decoupling of money market rates from the ECB's minimum bid rate due to underbidding in the main refinancing operations. Changing the maturities to better decompose timing and jump news at the monthly frequency would come at the cost that the timing news (which may have been more meaningful at fortnightly frequencies) observed in the pre-2001 period would be lost.
} 
nounced separately from the more detailed communication, statistical methods are not necessarily needed to extract the nature of policy news. By the time the ECB's press conference starts, the actual interest rate decision has already been known for three quarters of an hour. Changes in forward rates during the press conference can therefore only be due to communication news, and cannot be contaminated by jump and timing components.

As a result, a viable alternative for decomposing information is to segregate the time windows into decision and communication windows. The first window, spanning from 13:35-14:05 will be used to construct jump and timing news that relate to the interest rate decision. We define $j u m p_{t}^{D} \equiv \Delta f_{t, D}^{30}$ and obtain timing $g_{t}^{D}$ again as the residual from the regression:

$$
\Delta f_{t, D}^{10}=\alpha_{0}^{*}+\alpha_{1}^{*} j u m p_{t}^{D}+\operatorname{timing}_{t}^{D}
$$

with $D$ denoting the decision window. The $\bar{R}^{2}$ of this regression is 0.67 . The second window, from 14:20-15:50, is used to get a measure of the path news, by defining $\Delta f_{t, C}^{150} \equiv$ pat $h_{t}^{C}$, with $C$ denoting the communication window.

There exist days on which no press conference was held, and hence, in effect, no path news are measured. There are 17 such occasions in our sample, mainly concentrated in the preNovember 2001 period. To deal with this issue, we have simply set the path news equal to zero on such days.

\subsection{Jobless claims}

The release of US weekly jobless claims occurs at 14:30 every Thursday, coinciding with the beginning of the ECB's press conference. We therefore test for the possibility that the weekly jobless claims influence the construction of indicators of monetary policy news. For the rotated factors obtained from principal component analysis, we regress $z_{1}$ and $z_{2}$ on the jobless claims variable (defined as deviations of the data release from market expectations). This results in insignificant coefficients of negligible size. ${ }^{7}$ For the recursive regressions, we include the same jobless claims variable as a control variable in the regressions in (2) to (4). This results again in insignificant coefficients of negligible size for the jobless claims variable. We thus decided to exclude the jobless claims variable in the computation of the surprise measures. More detailed results are available upon request from the authors.

\section{Evolution of monetary policy news over time}

\subsection{Main results}

The three sets of multidimensional indicators of monetary policy news are shown in Figure 3. Panel (a) in the top of Figure 3 shows the indicators obtained from the structural principal components analysis and panel (b) in the middle of Figure 3 shows the indicators from the recursive

\footnotetext{
${ }^{7}$ We have also inspected cross-plots of $z_{1}$ and $z_{2}$ against jobless claims with a superimposed non-parametric estimate of the relation, but find no evidence of non-linearity.
} 
regressions, both based on the wide time window. Panel (c) at the bottom shows the indicators obtained from the two narrow decision and communication windows. Positive entries indicate an unanticipated tightening of money market rates (in percentage points) in the respective dimension: jump (and if applicable timing) at the short end of the money market yield curve and path, relating to changes in expectations of the course of monetary policy several months out.

On average the size of monetary policy news is relatively small, with the average of the absolute surprise ranging from 1 to 3 basis points. The size of the news, especially in terms of the jump (and timing) dimension, declines over time, with the largest news clearly concentrated in the first two years of the sample. At the same time, from the beginning of 2002 onwards path news appear to have become relatively more important.

Comparing the news derived from the different methods to one another there are clearly more similarities than differences. For example, the jump news from the principal component analysis is positively correlated with both the jump and timing news from the recursive regressions (with significant correlation coefficients of 0.68 and 0.64 respectively). Jump and timing news are also closely related to a traditional monetary policy news measure constructed from daily 1 month EURIBOR data (with correlation coefficients ranging from 0.45 to 0.74 ).

The path news obtained from the indirect statistical methods are closely related to path news derived directly from the narrow press conference window. This can be seen in Figure 4, where path news from factor analysis (top panel) and recursive regressions (bottom panel) are plotted against the news obtained from the narrow windows, respectively. The correlation between path news derived from the recursive regressions and path news derived directly from the narrow window is high (0.79) and somewhat larger than the respective correlation between news derived from the factor analysis and news obtained from the narrow window (0.62). Furthermore, in most cases path news obtained from econometric methods are very small when no press conference took place (and news from the narrow window are set to zero). We thus conclude that the statistical methods for constructing communication news of monetary policy appear to be largely valid.

\subsection{Interpreting specific events}

The measures of monetary policy news are consistent with a narrative approach to specific episodes informed by knowledge about monetary policy and the macroeconomic circumstances. An example of an episode where the three different measures consistently bear out the same nature of news is the ECB's 25 basis point interest rate cut of 10 May 2001, which had not been priced in by financial markets. Figure 5 displays the money market yield curve as at 13:35 and 14:05, before and after the announcement of the decision, and at 15:50, after the end of the press conference. The parallel downward shift in the money market curve following that decision (see Figure 5) is consistently borne out as jump news by all three methods. As evident from Figure 5, this adjustment took place within the time of the "decision window". The money market curve did not move during the "communication window", suggesting that market expectations of the path of monetary policy remained unaltered on the basis of the information provided during the ECB's press conference.

Between June 2003 and November 2005 there have been no changes in the ECB policy inter- 
est rate. While the markets do not appear to have been surprised by the decisions themselves during this time period, the measures suggest some readjustments of expectations about the future path of interest rates in response to ECB communication. For example, in spring 2004 data indicating only modest growth in euro area economic activity and a number of shocks (including for example, exchange rate volatility and the March 11 terrorist attacks in Madrid) led markets to attach a higher probability to interest rate cuts at some point, although no immediate change was expected at the Governing Council meeting of April 2004. In fact, policy interest rates remained unchanged at that meeting, but the Introductory Statement to the press conference stated that the monetary policy stance remained in line with price stability. This triggered an upward shift in the money market curve at longer maturities (see Figure 6) and is consistently borne out by positive path news across all three methods.

While the different indicators provide largely consistent information about monetary policy, the exercise illustrated in Figure 4 identifies very few occasions when the indicators are not fully aligned. The period around early 2001, marking the beginning of a series of cuts in monetary policy rates, was characterised by relatively large volatility in measured news. Against market expectations, there was no interest rate cut on 11 April 2001. The changes in interest rates nevertheless appear to suggest that markets expected an interest rate cut which was not delivered. In particular, the tone of the press conference was perceived as signalling a "wait and see" attitude by the ECB, leading to an upward revision of expectations about future rates in monetary policy. For this instance, while the three different indicators bring out the changes in the money market curve as "tightening news", they are associated to timing news on the basis of the recursive regression method (as well as the separation of the time windows) and to jump on the basis of the principal components method.

The spring 2001 episode is one of very few instances where the different methods do not result in conclusive messages about the exact nature of monetary policy news. Figure 7 shows that money market rates of shorter maturities were largely detached from the level of the ECB's minimum bid rate which is indicated by the horizontal line. In fact, the episode falls into a period when especially the short end of the money market yield curve was affected by underbidding in the ECB's main refinancing operations. A similar episode occurred in March 2003. The impact of underbidding on money market interest rates varied across maturities. Since the indicators do not build on the same information set, but rely on yields of different maturities, on these very few occasions the results are not fully conclusive across methods.

Overall, the indicators proposed in section 3 point to the same qualitative nature of news about monetary policy on ECB Governing Council meeting days. Furthermore, the qualitative description of changes in policy expectations revealed by the indicators matches a judgemental assessment - informed by macroeconomic developments and the substance of ECB communication - of market developments. Therefore, the indicators provide a promising tool for extracting news about monetary policy based on an objective, reproducible method, without the need to judge the precise substance and meaning of central bank communication. 


\section{Impact of monetary policy news on longer-term yields}

To assess the impact of the extracted news components of monetary policy on yields, we run the following regressions

$$
\Delta y_{t}^{\tau}=\boldsymbol{\beta}^{\prime} \mathbf{I}_{t}+\varepsilon_{t}
$$

where $\Delta y_{t}^{\tau}$ is the change in the $\tau$ days ahead yield, $\forall \tau=60,70,80, \ldots, 3650$, viz, over the long time window, including the decision and press conference. Including a constant, $\mathbf{I}_{t}$ consists of the changes in the news that were extracted using the previously described methods in section 3. Once again, since US jobless claims are released within the same time interval, we included jobless claims as a control variable in $\mathbf{I}_{t} . \mathbf{I}_{t}$ thus takes the from $\mathbf{I}_{t}=$ $\left(1, \text { jump }_{t}, \text { timing }_{t}, \text { path }_{t}, \text { jobless }_{t}\right)^{\prime}$. As the impact of jobless claims data on $\Delta y_{t}^{\tau}$ is negligible and statistically insignificant, we do not report the results here. ${ }^{8}$

As was shown earlier, the elements of $\mathbf{I}_{t}$ are constructed variables. When computing standard errors it thus becomes important to account for the extra sample variation that comes through this construction. We account for this variation in two different ways. First, for the principal components based method we follow Gürkaynak et al. (2005) and resort to resampling techniques, ie., we use a non-parametric bootstrap to compute confidence bounds for $\beta$ in (5) (Gürkaynak et al., 2005). ${ }^{9}$ The results from regressing jump and path news (based on the principal-components indicators) on $\Delta y_{t}^{\tau}$ for maturities $\tau=60,70,80, \ldots, 3650$ with bootstrapped $95 \%$ confidence intervals computed as the $2.5 \%$ and $97.5 \%$ percentiles from 10000 draws are shown in Figure 8. Second, for the sequential regressions in (2), (3) and (5), we derive asymptotic confidence bands. The sequential regressions can be thought of as a sequential Method of Moments (MOM) estimation problem and it is possible to derive the correct analytic asymptotic variance of $\beta$, taking account of the multiple steps in the estimation. ${ }^{10}$ The derivation is shown in the Appendix. Figure 9 below shows the estimates of the effects of jump, timing and path news on $\Delta y_{t}^{\tau}$ for maturities, $\tau=60,70,80, \ldots, 3650$ together with asymptotic $95 \%$ confidence bounds. Finally, we present the impact of monetary policy news on yield changes from the two narrow windows in Figure 10, also with the correct asymptotic $95 \%$ confidence bands. ${ }^{11}$

Overall the results show that the type of monetary policy news is crucial in distinguishing how monetary policy affects the yield curve across the maturity spectrum. In particular, jump news tend to have an impact on the short to medium term maturities. Timing news are mostly insignificant, except for the shortest maturities. Finally, irrespective of the method that is used

\footnotetext{
${ }^{8}$ The results regarding the impact of jobless claims on yield changes can be obtained from the authors upon request.

${ }^{9}$ Gürkaynak et al. (2005) actually draw from these data as if they were i.i.d, that is, homogenous, which could be hard to justify - as evident from Figure 3. We verified our results with a block bootstrap procedure to ensure that neighbouring news are drawn together so as to preserve the structure of the data over specific intervals. Drawing blocks of size three lead to adequate re-sampling properties. There were no noticeable differences in the size of the standard errors so that the results based on the i.i.d appear to be adequate.

${ }^{10}$ Note that for the method based on principal-components analysis above, it is, in principle, also possible to write down a set of moment conditions for the estimation of the factors. However, it is not clear how the rotation of the factors impacts on the variance. We thus resort to resampling techniques.

${ }^{11}$ Note that there are only two regression steps, rather then three, so that the derivation of the asymptotic variance is similar to that outlined in the Appendix. To preserve space, we do not report the derivation of this variance.
} 
to extract news about the future path of interest rates, path news have a significant impact across all maturities. The results consistently suggest that, depending on the precise maturity, $30-50 \%$ of changes in the path component of monetary policy news translate into changes in medium- to long-term yields. As these news mainly related to ECB communication during the press conference, these results point to a significant role for central bank communication in shaping the impact of monetary policy on financial markets.

We compare our results with evidence obtained for the US. Table 1 presents the impact of monetary policy news as obtained on the basis of the three methods for the euro area together with the results that Gürkaynak et al. (2005) and Gürkaynak (2005) obtained for the US. The coefficients for both currency areas - as regards both time profile and size - are consistent. This is especially the case for the principal components approach, where there is a close methodological match between our study and the results obtained in Gürkaynak et al. (2005).

\section{Conclusions}

We use high-frequency money market data on monetary policy days to construct multidimensional indicators of news about ECB monetary policy and study their impact on the euro area yield curve. Our analysis contributes in two ways to the existing literature on the financial market impact of monetary policy decisions and communication. First, we exploit the institutional feature that the ECB announces and explains its monetary policy decisions on two different time instances during the day of rate-setting meetings. This allows us to validate econometric methods decomposing changes in the money market yield curve over a wide time window (comprising decision and communication events) to indirectly construct indicators capturing the decision and the communication dimensions separately. Our results support the use of these indirect econometric methods in constructing measures of news from central bank communication. Indeed indicators of news related to ECB communication derived using different econometric methodologies give information that is very similar to the information from the direct approach. Second, we take a more comprehensive approach to analyse the financial market impact of the ECB's policy decisions and communication than in the current literature which is based on single-factor indicators and subjective measures of the tone of ECB statements. Although the ECB does not make explicit bias statements about future policy interest rates, our results suggest that market expectations of the path of monetary policy may change considerably during the ECB's press conference. Moreover, it appears that these changes have a significant and sizeable impact on medium to long-term interest rates, while immediate policy decisions have an impact only at shorter maturities. In addition, the size of the impact on yields in the euro area is similar to that measured for US data. This would suggest that the possible differences between communication practices of the ECB and the Federal Reserve in terms of how explicit "forward-looking" statements about upcoming decisions are used do not imply differences in how financial markets have reacted to policy announcements.

Our results show that news stemming from the ECB's communication matter more for longterm interest rates - crucial for the transmission of monetary policy - than news about policy decisions. Consistency between central bank communication and decisions is therefore important. The evidence presented in this paper suggests that ECB words and actions have indeed been consistent - otherwise markets would not have reacted to communication events in the first place. 


\section{References}

Andersson, M., L. Hansen, And S. Sebestyen (2006): "The Effects of Macroeconomic and Monetary Policy Announcements on the Euro Area Intraday Bond Market." ECB Working Paper No. 631.

BAI, J. AND S. NG (2002): “Determining the Number of Factors in Approximate Factor Models." Econometrica, 70, 191-221.

Bernoth, K. AND J. VON HAgen (2004): “The Euribor Futures Market: Efficiency and the Impact of ECB Policy Announcements." International Finance, 7, 1-24.

Brousseau, V. (2002): “The functional form of yield curves." Working Paper No. 148, ECB.

(2006): “The Spectrum of Euro-Dollar." in Long Memory in Economics, edited by G. Teyssièrre and A. Kirman, Heidelberg:: Springer Verlag.

CRAGG, J. G. AND S. G. DONALD (1997): "Inferring the Rank of a Matrix." Journal of Econometrics, 76, 223-50.

Ehrmann, M. And M. Fratzscher (2003): “Monetary Policy Announcements and Money Markets: A Transatlantic Perspective." International Finance, 6, 309-328.

_ (2005): “How Should Central Banks Communicate?” Working paper No. 557, ECB.

(2006): “Communication and Decision-Making by Central Bank Committees: Different strategies, same effectiveness?" Journal of Money, Credit and Banking, forthcoming.

GERLACH, S. (2004): “Interest Rate Setting By the ECB: Words and Deeds.” Discussion Paper No. 4775, CEPR.

GIES, M. (2005): “Do ECB's Statements Steer Short-Term and Long-Term Interest Rates in the Euro-Zone?" Unpubplished Manuscript.

GÜRKAYNAK, R. S. (2005): "Using federal funds futures contracts for monetary policy analysis." Mimeo.

GÜRKAYNAK, R. S., B. SACK, AND E. T. SWANSON (2005): “Do Actions Speak Louder Than Words? The Response of Asset Prices to Monetary Policy Actions and Statements." International Journal of Central Banking, 1, 55-93.

Hartmann, P., M. Manna, And M. Manzanares (2001): "The Microstructure of the Euro Money Market." Journal of International Money and Finance, 20, 895-948.

HeinemanN, F. And K. Ullrich (2005): "Does it Pay to Watch Central Bankers Lips? The Information Content of ECB Wording." Working Paper No. 05-70, ZEW.

Meyer, T. (2004): “The Risks to Price Stability." Deutsche Bank Research Focus Europe, 19 July 2004.

NEWEY, W. K. (1984): “A Method of Moments Interpretation of Sequential Estimators." Economics Letters, 14, 201-206. 
Newey, W. K. And K. D. West (1987): “A Simple, Positive Semi-Definite, Heteroskedasticity and Autocorrelation Consistent Covariance Matrix." Econometrica, 55, 703-708.

PAGAN, A. R. (1984): "Econometric Issues in the Analysis of Regressions with Generated Regressors." International Economic Review, 25, 221-247.

(1986): "Two Stage and Related Estimators and their Applications." Review of Economic Studies, 53, 517-538.

Perez-Quiros, G. AND J. SiCIlia (2002): "Is the European Central Bank (and the US Federal Reserve) Predictable?" Working Paper No. 192, ECB.

RosA, C. AND G. Verga (2005): "The Importance of the Wording of the ECB." Discussion Paper No. 694, CEP.

SEbestyÉn, S. (2006): “What Drives Money Market Rates?" Unpublished Manuscript.

SEBESTYÉN, S. AND J. SiCILIA (2005): “The Impacts of the External Communication of the ECB on Financial Markets in the Euro Area." Unpublished Manuscript.

Wilhelmsen, B.-R. AND A. ZAGHini (2005): “Monetary Policy Predictability in the Euro Area: An International Comparison." Working Paper No. 504, ECB.

Woodford, M. (2003): Interest and Prices: Foundations of a Theory of Monetary Policy, Princeton University Press. 


\section{Appendix}

\section{A1. Factor rotation}

The notation regarding the rotation of the factors follows the outline given in Gürkaynak et al. (2005) on pages 90 to 91 . Let the factors $F$ be related to another set of factors $Z$ through the following relationship

$$
Z=F U
$$

where

$$
U=\left[\begin{array}{ll}
\alpha_{1} & \beta_{1} \\
\alpha_{2} & \beta_{2}
\end{array}\right]
$$

and $U$ is an orthogonal matrix. $U$ is identified by a number of restrictions. These are, unit length of the columns of $U$, orthogonality of the columns of $Z=\left(z_{1}, z_{2}\right)$, ie. $E\left(z_{1} z_{2}\right)=0=$ $\alpha_{1} \beta_{1}+\alpha_{2} \beta_{2}$. We also need the restriction that $z_{2}$ does not influence (load into) $\Delta f_{t}^{10}$, so that we set $\gamma_{2} \alpha_{1}-\gamma_{1} \alpha_{2}=0$, where $\gamma_{1}$ and $\gamma_{2}$ are entries $(1,1)$ and $(2,1)$ in the $2 \times N$ matrix $\Omega^{\prime}$, viz, the loadings of $\Delta f_{t}^{10}$ on the two factors $f_{1}$ and $f_{2}$. Also, $z_{1}^{*}$ and $z_{2}^{*}$ are rescaled so that $z_{1}$ moves $\Delta f_{t}^{10}$ one to one and $z_{2}$ moves $\Delta f_{t}^{360}$ one to one.

A preliminary set of unstandardised but rotated factors are then computed as

$$
\begin{aligned}
& z_{1}^{*}=\alpha_{1} f_{1}+\alpha_{2} f_{2} \\
& z_{2}^{*}=\beta_{1} f_{1}+\beta_{2} f_{2}
\end{aligned}
$$

with

$$
\begin{aligned}
& \alpha_{1}=\frac{\gamma_{1}}{\gamma_{1}+\gamma_{2}} \\
& \alpha_{2}=\frac{\gamma_{1}}{\gamma_{1}+\gamma_{2}} \\
& \beta_{1}=\frac{-\alpha_{2} \operatorname{Var}\left(f_{2}\right)}{\alpha_{1} \operatorname{Var}\left(f_{1}\right)-\alpha_{2} \operatorname{Var}\left(f_{2}\right)} \\
& \beta_{2}=\frac{\alpha_{1} \operatorname{Var}\left(f_{1}\right)}{\alpha_{1} \operatorname{Var}\left(f_{1}\right)-\alpha_{2} \operatorname{Var}\left(f_{2}\right)}
\end{aligned}
$$

These are then standardised according to $z_{1}=\xi_{1} z_{1}^{*}$ and $z_{2}=\frac{\zeta_{2}}{\zeta_{1}} z_{2}^{*}$ where $\xi_{1}, \zeta_{2}$, and $\zeta_{1}$ are coefficient estimates from the regressions

$$
\begin{aligned}
\Delta f_{t}^{10} & =\xi_{0}+\xi_{1} z_{1}^{*}+\epsilon_{1, t} \\
\Delta f_{t}^{360} & =\zeta_{0}+\zeta_{1} z_{1}^{*}+\zeta_{2} z_{2}^{*}+\epsilon_{2, t}
\end{aligned}
$$

so that we finally obtain $Z=\left(z_{1}, z_{2}\right)$. 


\section{A2. Asymptotic variance}

To illustrate the derivation of the asymptotic variance of $\beta$ we will, for reasons of simplicity, use the following notation. We define $v_{t}$ and $s_{t}$ as respectively timing $g_{t}$ and $p a t h_{t}$ news, and then use the following compact notation on equations (2) and (3)

$$
\begin{aligned}
\Delta f_{t}^{10} & =\alpha^{\prime} \mathbf{x}_{1 t}+v_{t} \\
\Delta f_{t}^{150} & =\gamma^{\prime} \mathbf{x}_{2 t}+s_{t}
\end{aligned}
$$

Also, $\mathbf{x}_{1 t}$ and $\mathbf{x}_{2 t}$ are defined as $\mathbf{x}_{1 t}=\left(1, \Delta f_{t}^{30}\right)^{\prime}$ and $\mathbf{x}_{2 t}={ }^{\prime}$, with $\boldsymbol{\theta}=\left(\boldsymbol{\alpha}^{\prime}, \boldsymbol{\gamma}^{\prime}\right)^{\prime}$ being the set of first stage estimation parameters corresponding to $\mathbf{x}_{1 t}$ and $\mathbf{x}_{2 t}$. Note that $\Delta f_{t}^{30}$ was given the jump news label earlier. Writing (5) compactly as

$$
\Delta y_{t}=\boldsymbol{\beta}^{\prime} \mathbf{x}_{3 t}+\varepsilon_{t}
$$

where, for this case $\mathbf{I}_{t}=\mathbf{x}_{3 t}=\left(1, \Delta f_{t}^{30}, v_{t}, s_{t}, j_{t}\right)^{\prime}$ with 1 being a vector of constants and $j_{t}$ a control variable for U.S. jobless claims data being released during the same time interval. One can get a consistent and asymptotically unbiased estimate of $\beta$ using OLS as long as the standard moment conditions of OLS are satisfied. We will assume that that is the case here. The problem, however, is to get the correct variance of $\boldsymbol{\beta}$, which, in general, will not be $E\left[\mathbf{x}_{3 t} \mathbf{x}_{3 t}^{\prime}\right]^{-1} \operatorname{Var}\left[\varepsilon_{t}\right]$, due to $v_{t}$ and $s_{t}$ being constructed variables, unless one can show that the expected value of the derivatives of the moment conditions of the last regression step in (14) is zero when taken with respect to the true parameter vector.

The problem can, in fact, be thought of as a standard sequential MOM estimation problem, as set out in Pagan (1984), Newey (1984) and Pagan (1986). The primary concern here is that $\boldsymbol{\alpha}$ and $\gamma$ in (12) and (13) respectively are unknown and are replaced by consistent estimates, and due to that, there is additional sample variation that comes into the estimation of $\beta$ in (14). Using the assumption of exogenous regressor, implicit to OLS regressions, one can formulate a standard set of moment conditions and think about deriving the asymptotic variance matrix of $\boldsymbol{\beta}$ within a MOM framework. Denoting $\mathbf{f}_{t}, \mathbf{g}_{t}$ and $\mathbf{h}_{t}$ the vectors of moment conditions for the regressions in equations (12), (13), and (14) respectively, one can show that the asymptotic variance matrix of $\beta$ takes the form

$$
\Sigma_{\beta}=\operatorname{Var}(\boldsymbol{\beta})+H_{\beta}^{-1} H_{\boldsymbol{\theta}}[\Psi] H_{\theta}^{\prime} H_{\beta}^{-1}
$$

where $H_{\boldsymbol{\beta}}=E\left[\frac{\partial \mathbf{h}_{t}}{\partial \boldsymbol{\beta}^{\prime}}\right], H_{\boldsymbol{\theta}}=E\left[\frac{\partial \mathbf{h}_{t}}{\partial \boldsymbol{\theta}^{\prime}}\right]$ and $P$ has the form

$$
\Psi=\left[\begin{array}{cc}
\operatorname{Var}(\boldsymbol{\alpha}) & \operatorname{Var}(\boldsymbol{\alpha}) G^{\prime} \\
\operatorname{Gar}(\boldsymbol{\alpha}) & \Sigma_{\boldsymbol{\gamma}}
\end{array}\right]
$$


where

$$
G=\left[\begin{array}{cc}
\gamma_{(3)} & 0 \\
0 & \gamma_{(3)} \\
0 & 0
\end{array}\right]
$$

and with

$$
\Sigma_{\gamma}=\operatorname{Var}(\boldsymbol{\alpha})+\operatorname{Gar}(\boldsymbol{\alpha}) G^{\prime}
$$

$\operatorname{Var}(\boldsymbol{\iota}) \forall \boldsymbol{\iota}=\boldsymbol{\alpha}, \boldsymbol{\beta}$ and $\boldsymbol{\gamma}$ is a Newey and West (1987) type heteroskedasticity and autocorrelation consistent (HAC) covariance matrix formed from the OLS regressions in (12) to (13) when ignoring the fact that we have constructed variables. Letting $\gamma_{(3)}$ denote the third element of $\gamma$, ie., the coefficient on $v_{t}$, and using this notation henceforth to denote element entries, it can be shown that $H_{\theta}$ takes the form

$$
H_{\boldsymbol{\theta}}=\left[\begin{array}{ccccc}
\vartheta & \vartheta E\left[\Delta f_{t}^{30}\right] & \beta_{(4)} & \beta_{(4)} E\left[\Delta f_{t}^{30}\right] & 0 \\
\vartheta E\left[\Delta f_{t}^{30}\right] & \vartheta E\left[\left(\Delta f_{t}^{30}\right)^{2}\right] & \beta_{(4)} E\left[\Delta f_{t}^{30}\right] & \beta_{(4)} E\left[\left(\Delta f_{t}^{30}\right)^{2}\right] & 0 \\
0 & 0 & 0 & 0 & \beta_{(4)} E\left[\nu_{t}^{2}\right] \\
0 & 0 & 0 & 0 & 0 \\
\vartheta E\left[j_{t}\right] & \vartheta E\left[j_{t} \Delta f_{t}^{30}\right] & \beta_{(4)} E\left[j_{t}\right] & \beta_{(4)} E\left[j_{t} \Delta f_{t}^{30}\right] & 0
\end{array}\right]
$$

with $\vartheta=\left(\beta_{(3)}-\beta_{(4)} \gamma_{(3)}\right)$ and one can recognise $H_{\boldsymbol{\beta}}=E\left[\frac{\partial \mathbf{h}_{t}}{\partial \boldsymbol{\beta}^{\prime}}\right]$ to be the cross-product moment matrix of $\mathbf{x}_{3 t}$.

The easiest way to derive the variance of $\beta$ to be as in (15) is in the following set up. Let $\boldsymbol{\phi}=\left(\boldsymbol{\theta}^{\prime}, \boldsymbol{\beta}^{\prime}\right)^{\prime}$ and $\boldsymbol{\theta}=\left(\boldsymbol{\alpha}^{\prime}, \boldsymbol{\gamma}^{\prime}\right)^{\prime}$ where $\boldsymbol{\alpha}, \boldsymbol{\gamma}$ and $\boldsymbol{\beta}$ are as defined above. For notational simplicity we will let $X_{t}$ represent all the relevant data entries. Also, we will in general suppress the dependence of the moment conditions on the data and parameter vectors and hence write, for example, $E\left[\mathbf{m}_{t}\right]$ instead of $E\left[\mathbf{m}_{t}\left(X_{t}, \boldsymbol{\phi}\right)\right]$. The following partitioned vector of moment conditions can then be formed

$$
E\left[\mathbf{m}_{t}\right]=E\left[\begin{array}{c}
\mathbf{f}_{t}\left(X_{t}, \boldsymbol{\alpha}\right) \\
\mathbf{g}_{t}\left(X_{t}, \boldsymbol{\theta}\right) \\
\cdots \\
\mathbf{h}_{t}\left(X_{t}, \boldsymbol{\phi}\right)
\end{array}\right]=\mathbf{0}
$$

where

$$
\begin{aligned}
\mathbf{f}_{t}\left(X_{t}, \boldsymbol{\alpha}\right) & =\left(v_{t}, \Delta f_{t}^{30} v_{t}\right)^{\prime} \\
\mathbf{g}_{t}\left(X_{t}, \boldsymbol{\theta}\right) & =\left(s_{t}, \Delta f_{t}^{30} s_{t}, v_{t} s_{t}\right)^{\prime} \\
\mathbf{h}_{t}\left(X_{t}, \boldsymbol{\phi}\right) & =\left(\varepsilon_{t}, \Delta f_{t}^{30} \varepsilon_{t}, v_{t} \varepsilon_{t}, s_{t} \varepsilon_{t}, j_{t} \varepsilon_{t}\right)^{\prime}
\end{aligned}
$$

From standard MOM theory we have that

$$
\sqrt{T}\left(\hat{\boldsymbol{\phi}}-\boldsymbol{\phi}_{0}\right) \stackrel{d}{\longrightarrow} \mathcal{N}\left(0, \boldsymbol{\Sigma}_{\boldsymbol{\phi}}\right)
$$


where $\Sigma_{\boldsymbol{\phi}}=\left(M^{-1} \Omega M^{-1 \prime}\right)$ with $M=\lim _{t \rightarrow \infty} T^{-1} E\left[\sum_{t=1}^{T} \mathbf{m}_{\phi^{t}}\right]$ and $\Omega=\operatorname{Var}\left(T^{-1 / 2} \sum_{t=1}^{T} \mathbf{m}_{t}\right)$. We will assume here that the moment conditions hold for all $t$ so that $T^{-1} E\left[\sum_{t=1}^{T} \mathbf{m}_{\phi} t\right]=$ $E\left[\mathbf{m}_{\phi^{t}}\right]$ which can then be written in the following partitioned form

$$
E\left[\mathbf{m}_{\phi t}\right]=E\left[\begin{array}{cccc}
\frac{\partial \mathbf{f}_{t}}{\partial \boldsymbol{\alpha}^{\prime}} & \frac{\partial \mathbf{f}_{t}}{\partial \boldsymbol{\gamma}^{\prime}} & \vdots & \frac{\partial \mathbf{f}_{t}}{\partial \boldsymbol{\beta}^{\prime}} \\
\frac{\partial \mathbf{g}_{t}}{\partial \boldsymbol{\alpha}^{\prime}} & \frac{\partial \mathbf{g}_{t}}{\partial \gamma^{\prime}} & & \frac{\partial \mathbf{g}_{t}}{\partial \boldsymbol{\beta}^{\prime}} \\
\cdots \cdots & \ldots & . & \cdots \\
\frac{\partial \mathbf{h}_{t}}{\partial \boldsymbol{\alpha}^{\prime}} & \frac{\partial \mathbf{h}_{t}}{\partial \gamma^{\prime}} & \vdots & \frac{\partial \mathbf{h}_{t}}{\partial \boldsymbol{\beta}^{\prime}}
\end{array}\right]=E\left[\begin{array}{cccc}
\frac{\partial \mathbf{f}_{t}}{\partial \boldsymbol{\alpha}^{\prime}} & \mathbf{0} & & \\
\frac{\partial \mathbf{g}_{t}}{\partial \boldsymbol{\alpha}^{\prime}} & \frac{\partial \mathbf{g}_{t}}{\partial \gamma^{\prime}} & \vdots & \mathbf{0} \\
\ldots & \ldots & \ldots & \ldots \\
\frac{\partial \mathbf{h}_{t}}{\partial \boldsymbol{\alpha}^{\prime}} & \frac{\partial \mathbf{h}_{t}}{\partial \gamma^{\prime}} & \vdots & \frac{\partial \mathbf{h}_{t}}{\partial \beta^{\prime}}
\end{array}\right]
$$

due to $\mathbf{f}_{t}$ being a function of $\boldsymbol{\alpha}$ only and $\mathbf{g}_{t}$ not being a function of $\beta . \Omega=\operatorname{Var}\left(T^{-1 / 2} \sum_{t=1}^{T} \mathbf{m}_{t}\right)$ can be computed using a standard Newey and West (1987) HAC covariance matrix. We can also partition $\Omega$ into

$$
\Omega=\left[\begin{array}{cccc}
\Omega_{f f} & \Omega_{f g} & \vdots & \Omega_{f h} \\
\Omega_{g f} & \Omega_{g g} & & \Omega_{g h} \\
\ldots & \ldots & . & \ldots \\
\Omega_{h f} & \Omega_{h g} & \vdots & \Omega_{h h}
\end{array}\right]
$$

where, for example, $\Omega_{f h}=\operatorname{Var}\left(T^{-1 / 2} \sum_{t=1}^{T}\left(\mathbf{f}_{t} \mathbf{h}_{t}^{\prime}\right)\right)$. Given the nature of the sequential regressions, we also know that, by construction of the first regression steps, $s_{t}$ and $v_{t}$ are orthogonal to $\varepsilon_{t}$ and are assumed uncorrelated over time as well, viz, $E\left[s_{t} \varepsilon_{t-j}\right]=0, \forall j>1$, we can set $\Omega_{g f}=\Omega_{h f}=\Omega_{h g}=0$, so that (22) is effectively block diagonal, taking the form

$$
\Omega=\left[\begin{array}{cccc}
\Omega_{f f} & \mathbf{0} & \vdots & \mathbf{0} \\
\mathbf{0} & \Omega_{g g} & & \\
\ldots \ldots & \ldots & . & \ldots \\
0 & \vdots & \Omega_{h h}
\end{array}\right]
$$

Given this partitioned set up, we can then follow Newey (1984) to find $\Sigma_{\beta}$ to be of the form

$$
\Sigma_{\beta}=H_{\beta}^{-1} \Omega_{h h} H_{\beta}^{-1 \prime}+H_{\beta}^{-1} H_{\theta}\left[F_{\theta}^{-1} \tilde{\Omega}_{f g} F_{\theta}^{-1 \prime}\right] H_{\theta}^{\prime} H_{\beta}^{-1}
$$

where $H_{\boldsymbol{\beta}}=E\left[\frac{\partial \mathbf{h}_{t}}{\partial \boldsymbol{\beta}^{\prime}}\right]$ and $H_{\boldsymbol{\theta}}=E\left[\frac{\partial \mathbf{h}_{t}}{\partial \boldsymbol{\theta}^{\prime}}\right]$ are again respectively the cross-product moment matrix of $x_{3, t}$ and as defined in (18), and $F_{\theta}$ and $\tilde{\Omega}_{f g}$ are respectively

$$
F_{\boldsymbol{\theta}}=E\left[\begin{array}{cc}
\frac{\partial \mathbf{f}_{t}}{\partial \boldsymbol{\alpha}^{\prime}} & \mathbf{0} \\
\frac{\partial \mathbf{g}_{t}}{\partial \boldsymbol{\alpha}^{\prime}} & \frac{\partial \mathbf{g}_{t}}{\partial \gamma^{\prime}}
\end{array}\right]
$$

and

$$
\tilde{\Omega}_{f g}=\left[\begin{array}{cc}
\Omega_{f f} & \mathbf{0} \\
\mathbf{0} & \Omega_{g g}
\end{array}\right]
$$


From the partitioned inverse of a matrix we know that

$$
\left[\begin{array}{cc}
\mathbf{A} & \mathbf{B} \\
\mathbf{C} & \mathbf{D}
\end{array}\right]^{-1}=\left[\begin{array}{ccc}
\mathbf{P}^{-1} & \vdots & -\mathbf{P}^{-1} \mathbf{B D}^{-1} \\
\ldots \ldots \ldots & \cdot & \ldots \ldots \ldots \ldots \ldots \ldots \ldots \\
-\mathbf{D}^{-1} \mathbf{C} \mathbf{P}^{-1} & \vdots & \mathbf{D}^{-1}+\mathbf{D}^{-1} \mathbf{C P}^{-1} \mathbf{B D}^{-1}
\end{array}\right]
$$

where $\mathbf{P}^{-1}=\mathbf{A}-\mathbf{B D}^{-1} \mathbf{C}$. Since in our case $\mathbf{B}=\mathbf{0}$, this then simplifies to $\mathbf{P}^{-1}=\mathbf{A}^{-1}$ and

$$
\left[\begin{array}{cc}
\mathbf{A} & \mathbf{0} \\
\mathbf{C} & \mathbf{D}
\end{array}\right]^{-1}=\left[\begin{array}{ccc}
\mathbf{A}^{-1} & \vdots & \mathbf{0} \\
\cdots \cdots \cdots & \cdot & \cdots \cdots \\
-\mathbf{D}^{-1} \mathbf{C A} & \vdots & \mathbf{D}^{-1}
\end{array}\right]
$$

Given the result in (26), the inverse of $F_{\theta}$ is then formed as

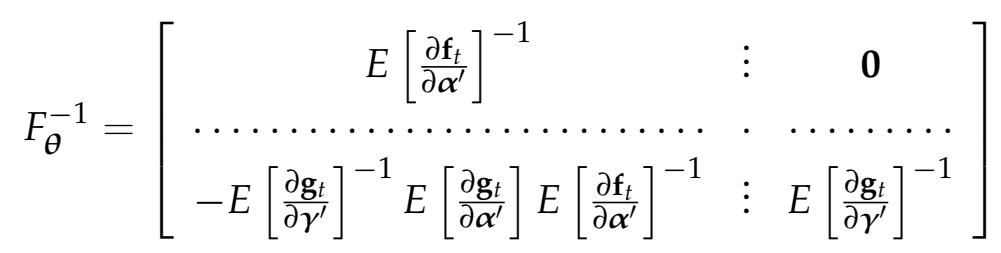

From (23),(25) and (27) we can then from

$$
\Sigma_{\beta}=H_{\beta}^{-1} \Omega_{h h} H_{\beta}^{-1 \prime}+H_{\beta}^{-1} H_{\theta}[\Psi] H_{\theta}^{\prime} H_{\beta}^{-1}
$$

with $\Psi$ taking on the form as given in (16)

$$
\Psi=\left[\begin{array}{cc}
\operatorname{Var}(\boldsymbol{\alpha}) & \operatorname{Var}(\boldsymbol{\alpha}) G^{\prime} \\
\operatorname{GVar}(\boldsymbol{\alpha}) & \Sigma_{\gamma}
\end{array}\right]
$$

To get this result, one needs to recognise that $E\left[\frac{\partial \mathbf{f}_{t}}{\partial \boldsymbol{\alpha}^{\prime}}\right]^{-1} \Omega_{f f} E\left[\frac{\partial \mathbf{f}_{t}}{\partial \alpha^{\prime}}\right]^{-1}$ and $E\left[\frac{\partial \mathbf{g}_{t}}{\partial \gamma^{\prime}}\right]^{-1} \Omega_{g g} E\left[\frac{\partial \mathbf{g}_{t}}{\partial \gamma^{\prime}}\right]^{-1}$ are just the standard HAC type covariance matrices of $\alpha$ and $\boldsymbol{\gamma}$, denoted respectively by $\operatorname{Var}(\boldsymbol{\alpha})$ and $\operatorname{Var}(\gamma)$ from the first and second step regressions, ignoring the fact that $v_{t}$ is a constructed variable in the second regression step. Also, $G$ is defined as

$$
G=-E\left[\frac{\partial \mathbf{g}_{t}}{\partial \gamma^{\prime}}\right]^{-1} E\left[\frac{\partial \mathbf{g}_{t}}{\partial \alpha^{\prime}}\right]
$$

which simplifies to (17) once these expectations are taken with respect to the true parameter vectors $\gamma$ and $\alpha$. 


\section{Figures and Tables}

Long time window: 13:35-15:50

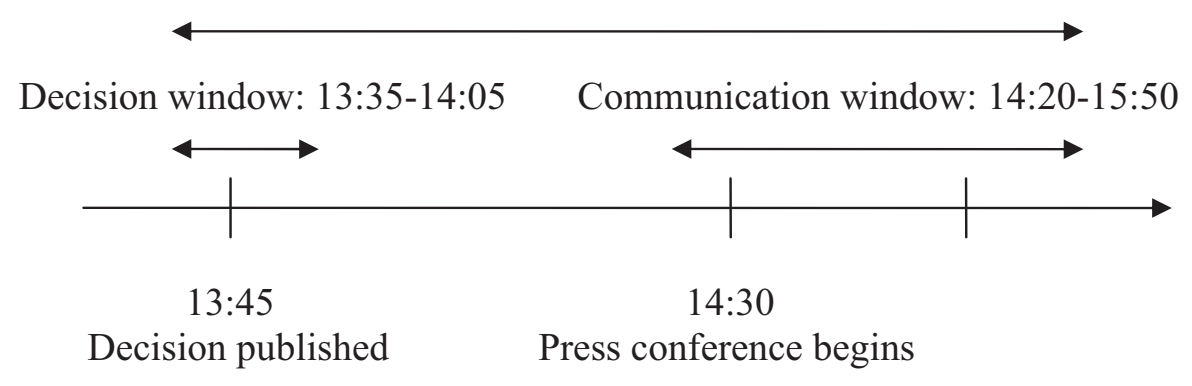

Figure 1: Graphical representation of the long, decision and communication time windows considered in the study.

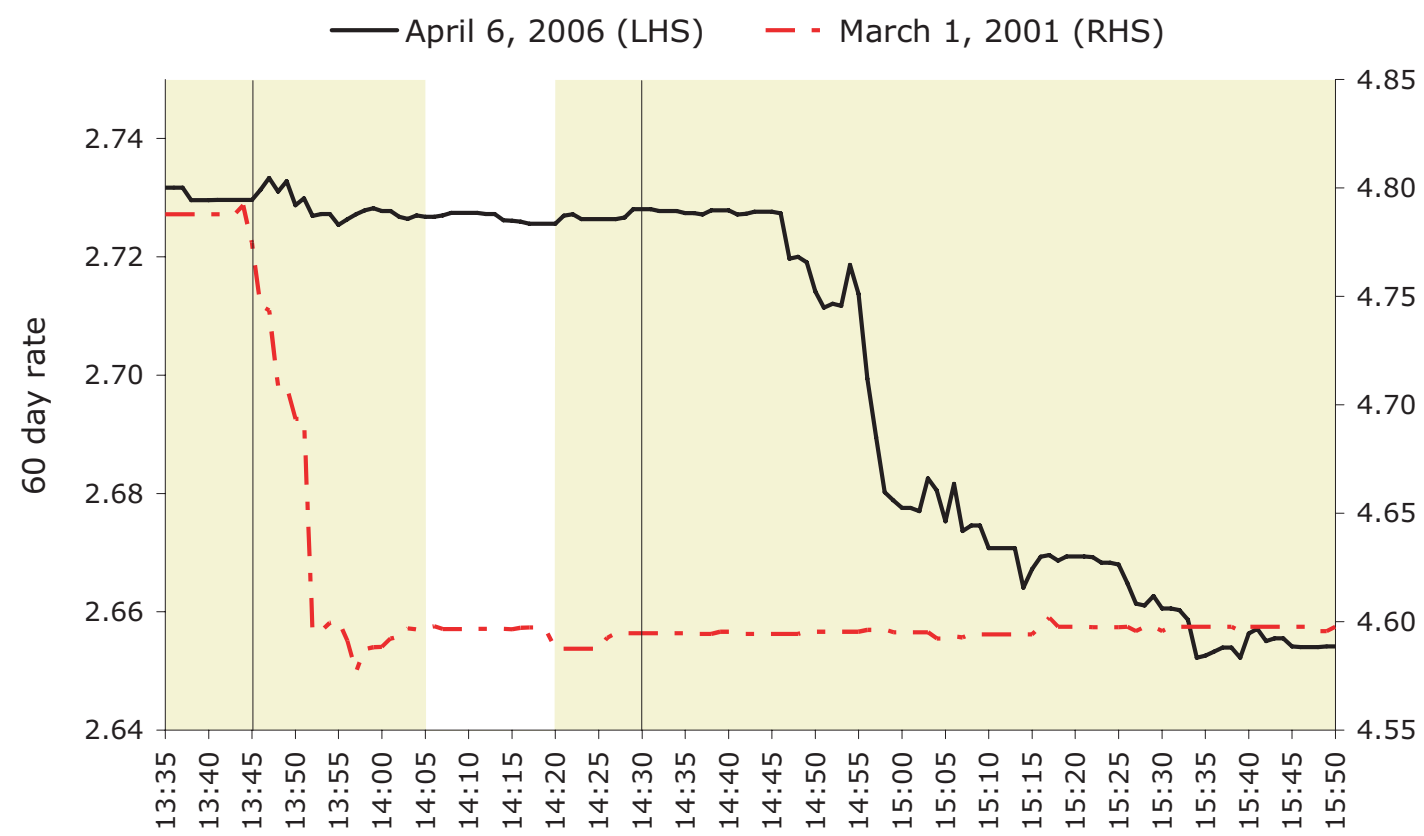

Figure 2: Intraday developments in the 60-day yield to maturity on 10 May 2001 and 6 April 2006. The vertical lines at 13:45 and 14:30 indicate the release of the monetary policy decision and the start of the ECB's press conference, respectively. The shaded areas indicate the "decision" and the "communication" windows. 


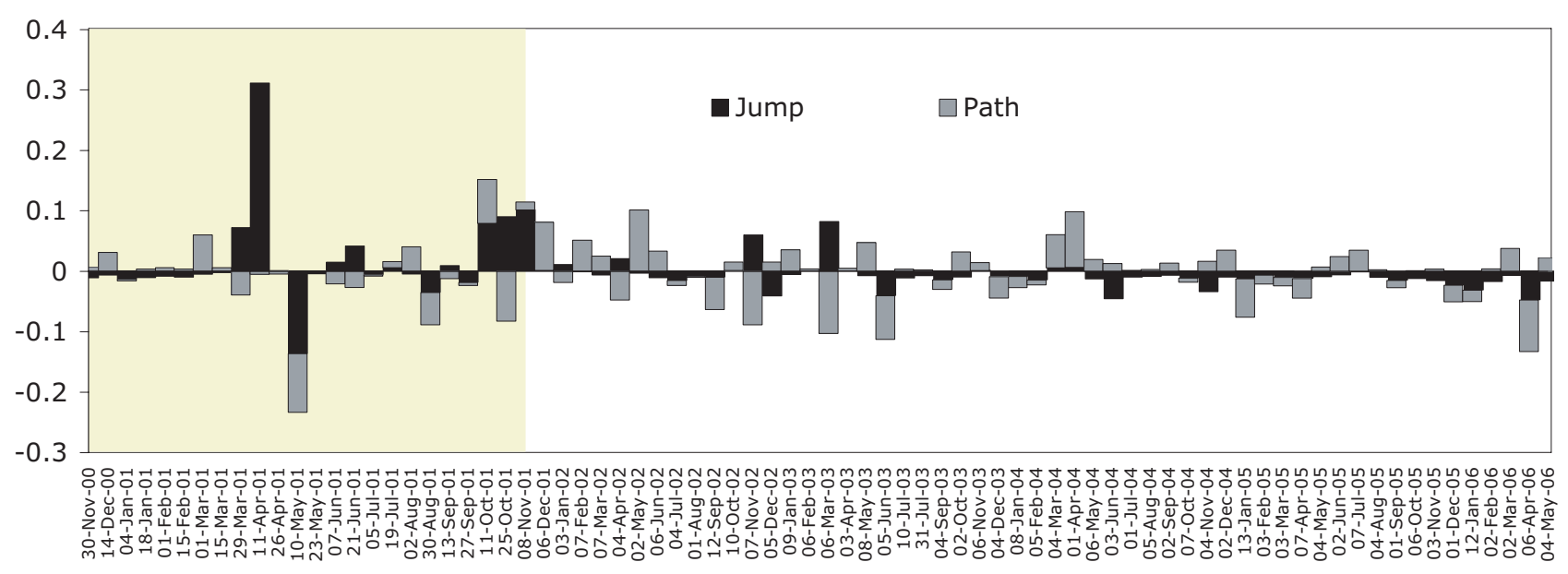

(a) Monetary Policy News extracted from rotated principal components

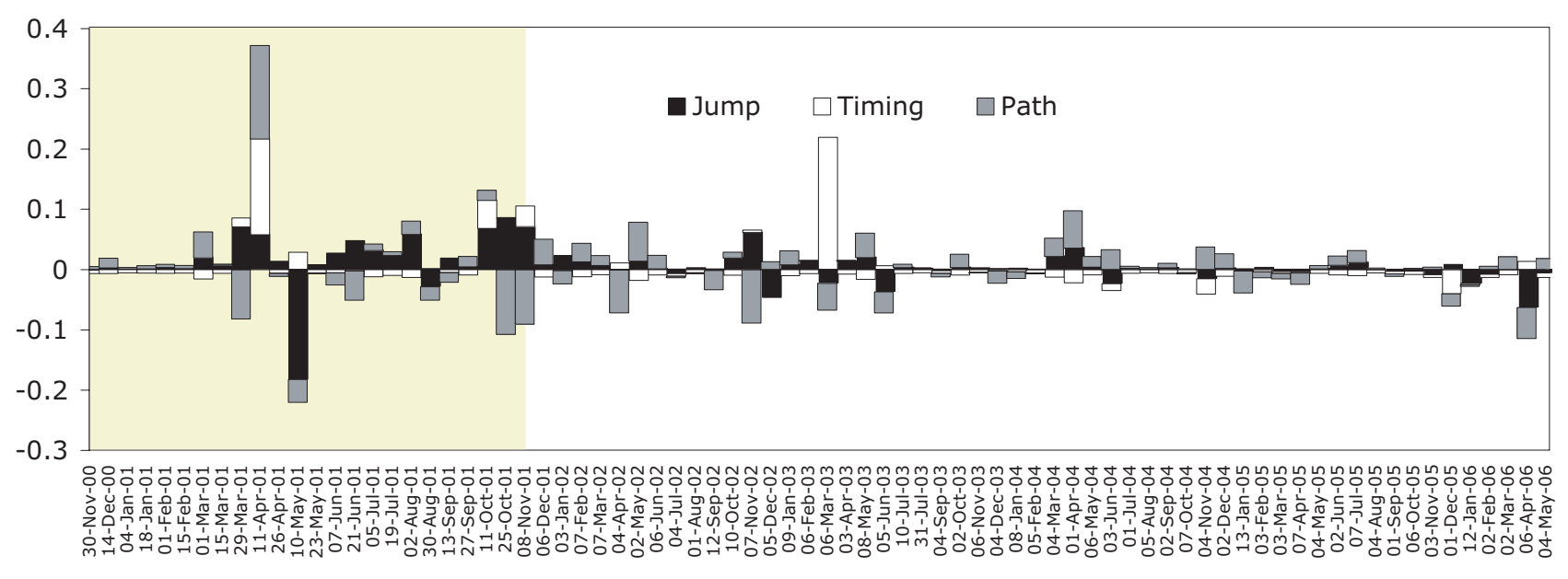

(b) Monetary Policy News extracted from recursive regressions

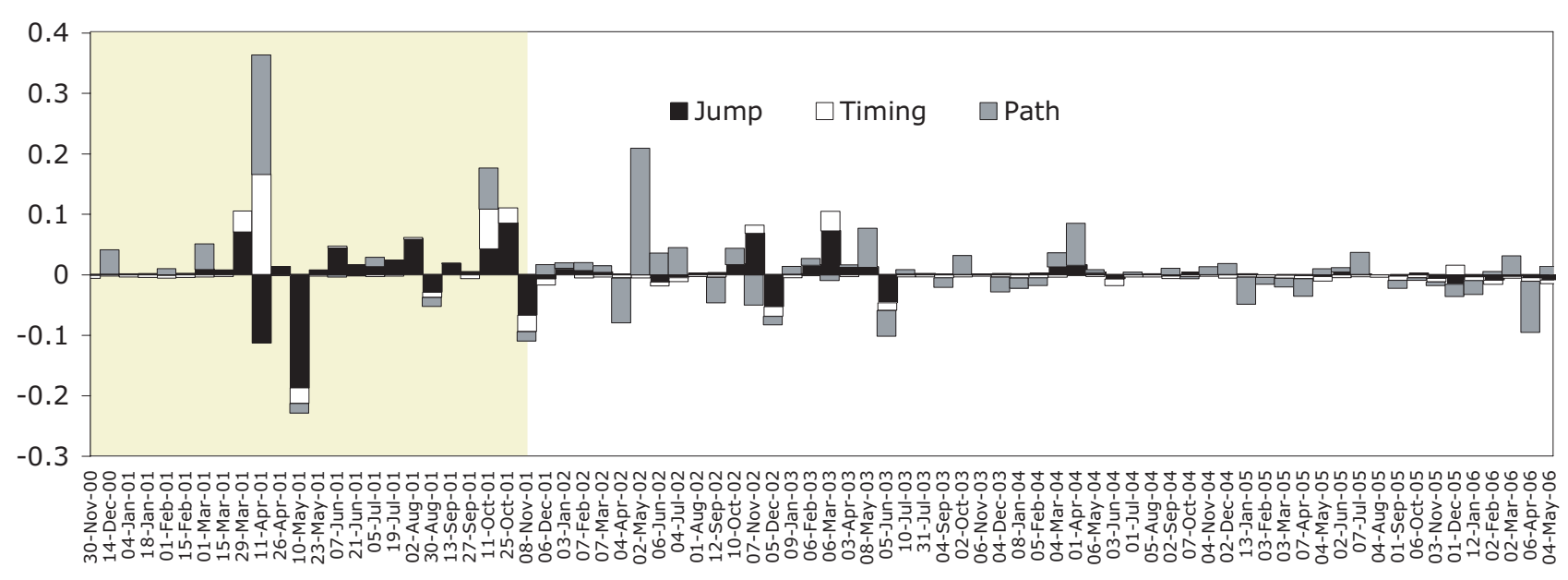

(c) Monetary Policy News extracted from decision and communication windows

Figure 3: Extracted jump, timing, and path news. Shaded area indicates fortnightly meeting frequency, except for 30 August 2001 meeting where there were 4 weeks between consecutive meetings. 


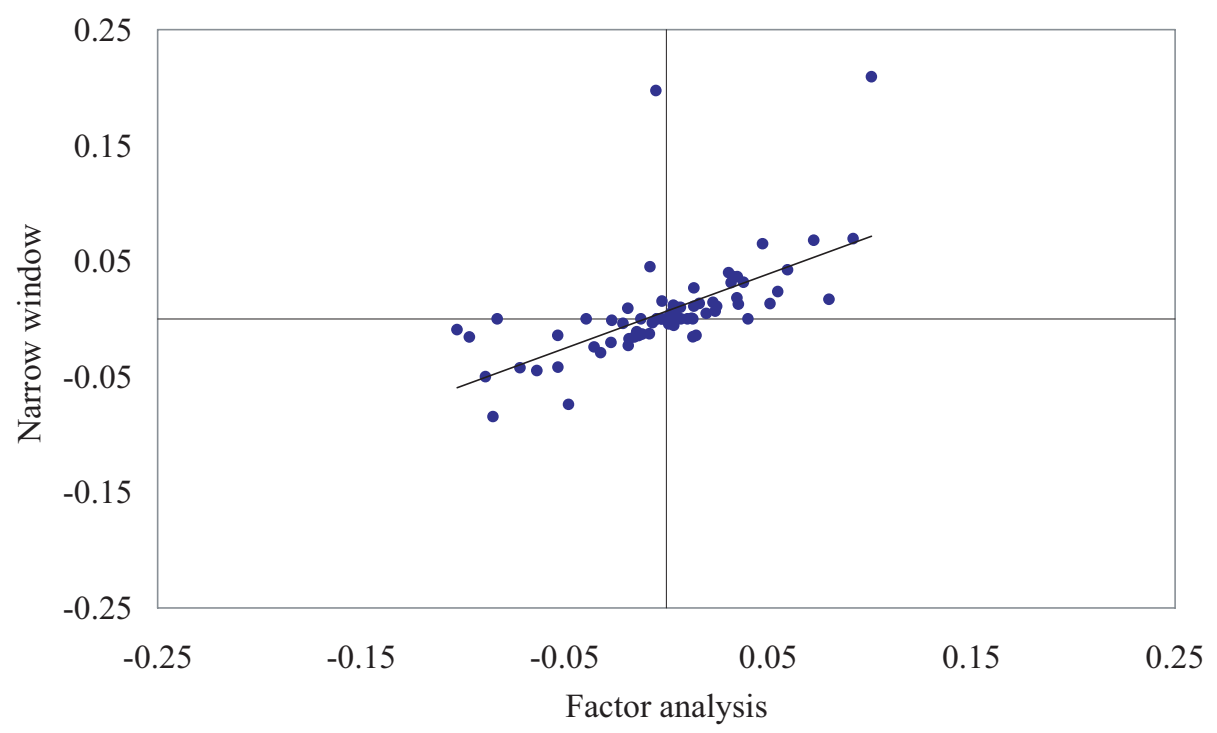

(a) Path from factor analysis against communication window

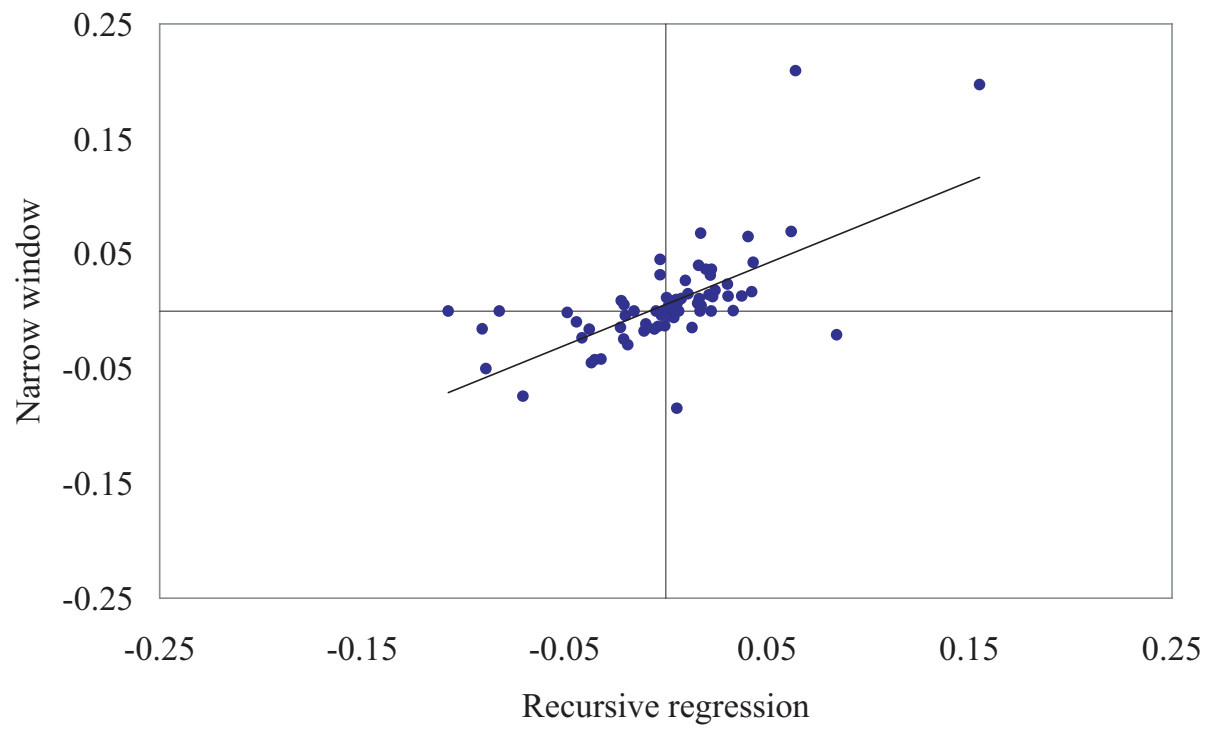

(b) Path from recursive regressions against communication window

Figure 4: Crossplot of path news across methods. 


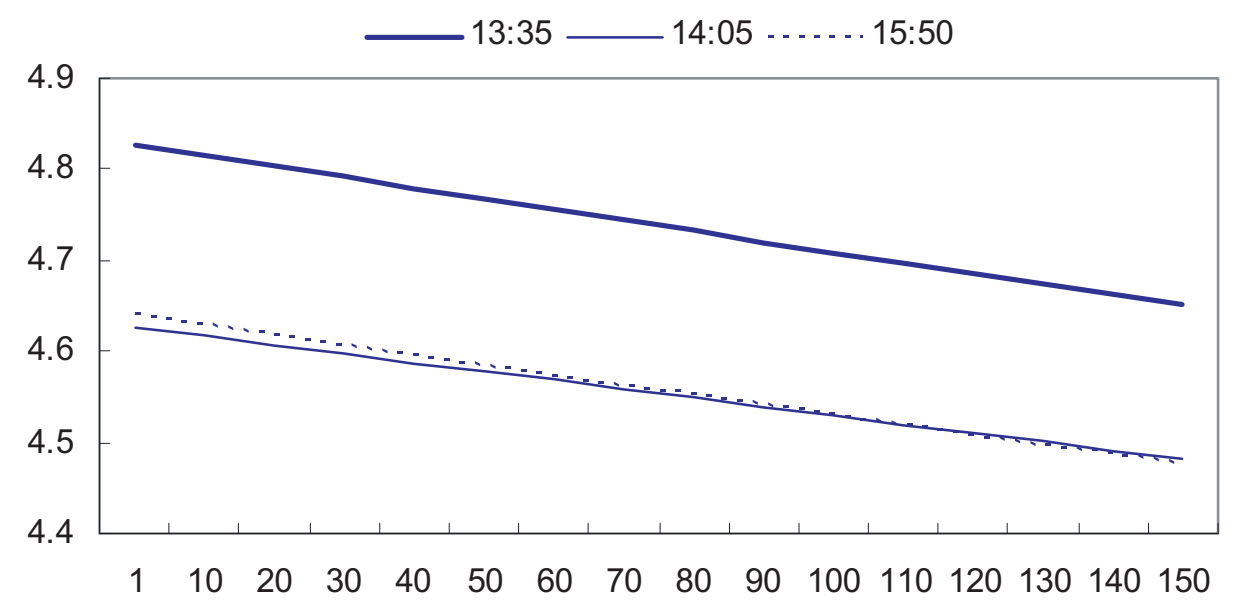

Figure 5: Money market rates as of 13:35, 14:05, 15:50 hours on 10 May 2001.

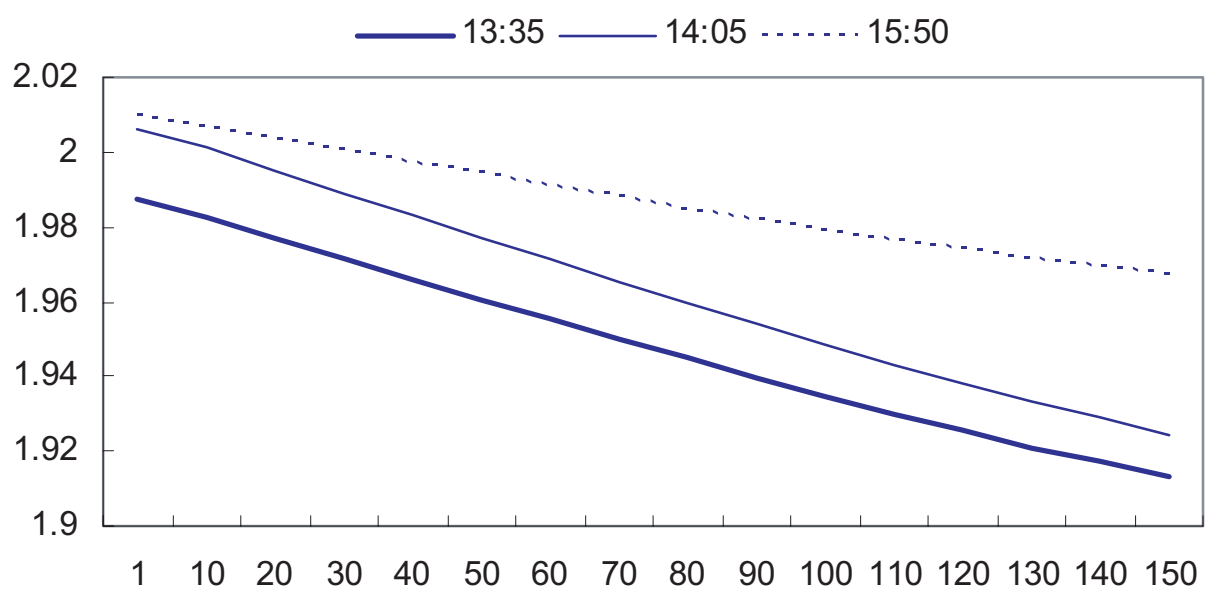

Figure 6: Money market rates as of 13:35, 14:05, 15:50 hours on 1 April 2004.

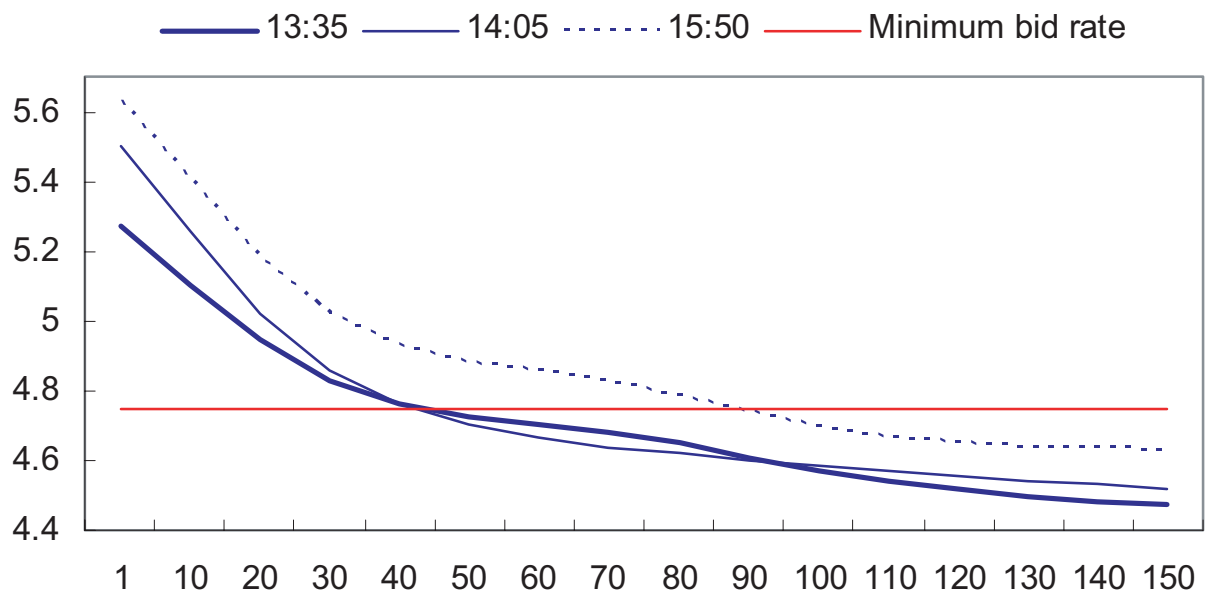

Figure 7: Money market rates as of 13:35, 14:05, 15:50 hours on 11 April 2001. 


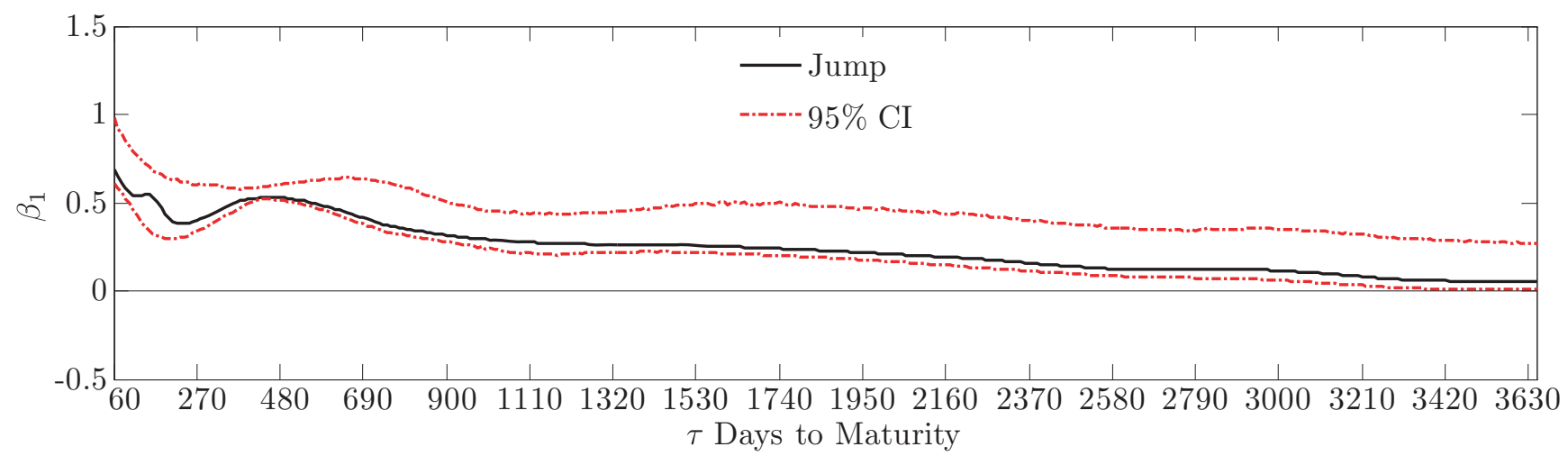

(a) Impact of the jump factor $z_{1}$ on changes in yields with bootstrapped confidence bounds

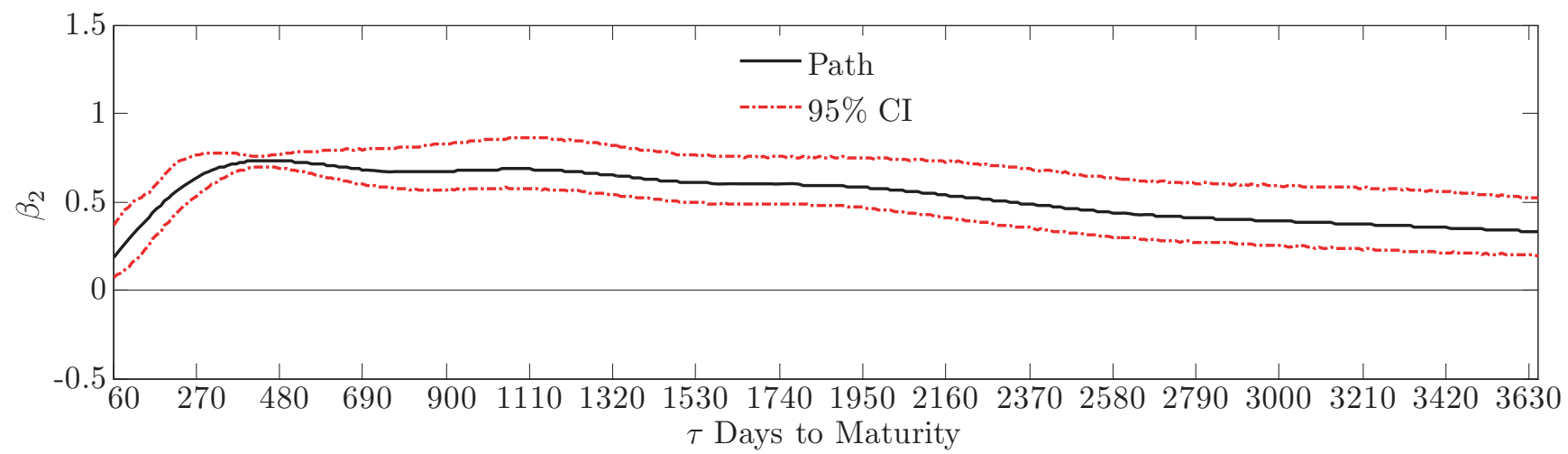

(b) Impact of the path factor $z_{2}$ on changes in yields with bootstrapped confidence bounds

Figure 8: Impact of news from the rotated factors from principal component analysis on yields at maturities $\tau=60,70,80, \ldots, 3650$ (based on wide time window). 


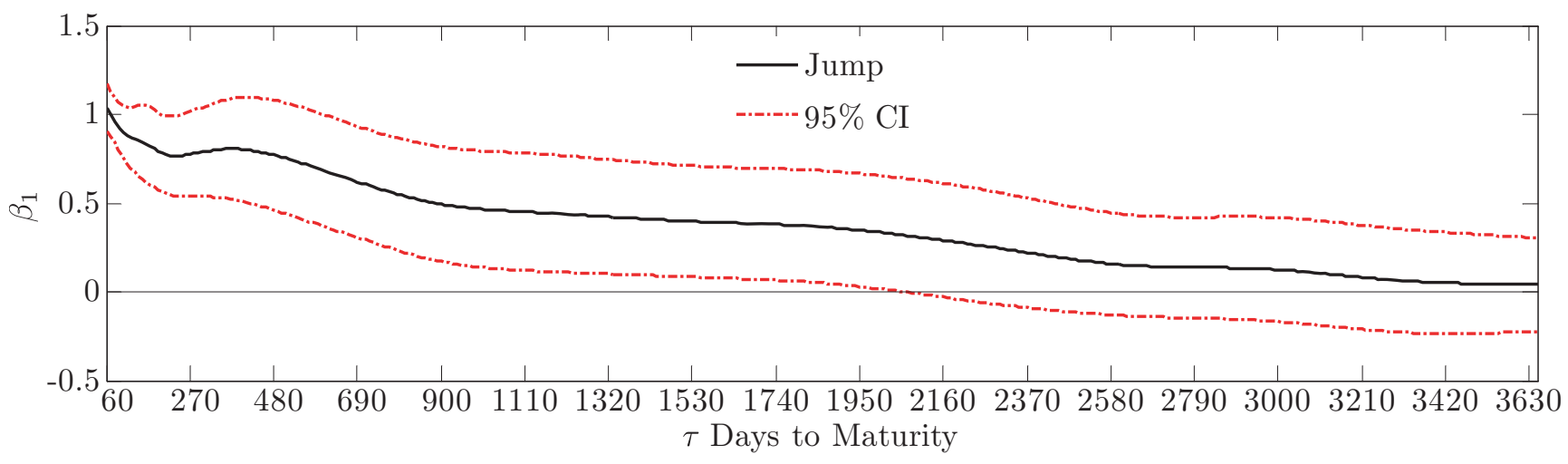

(a) Impact of jump news on changes in yields with corrected asymptotic $95 \%$ confidence bounds

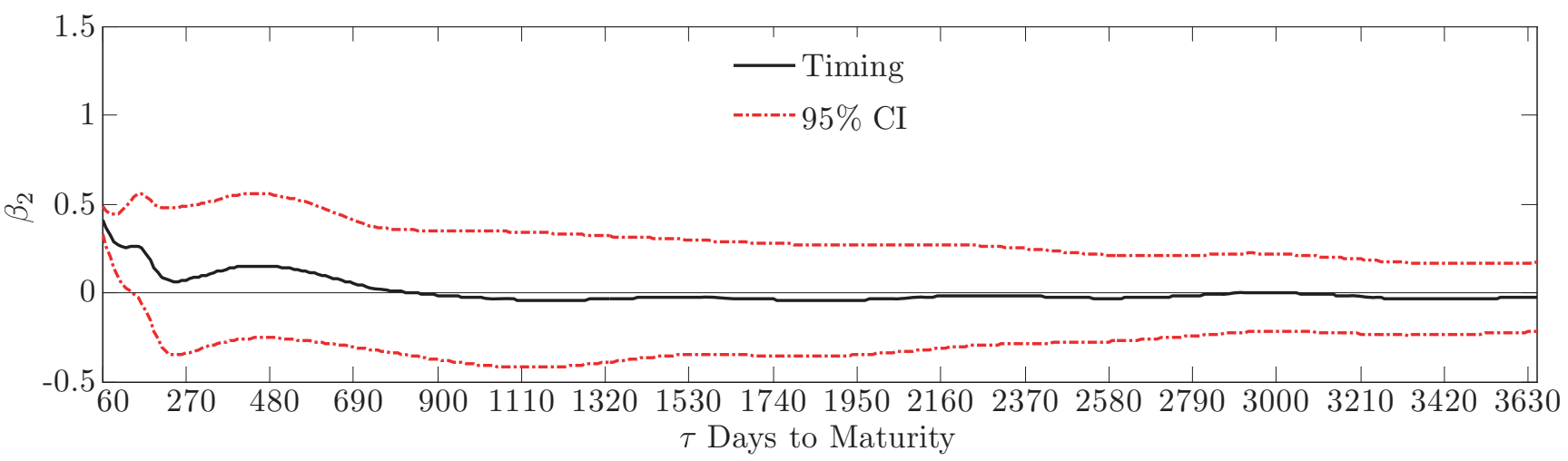

(b) Impact of timing news on changes in yields with corrected asymptotic 95\% confidence bounds

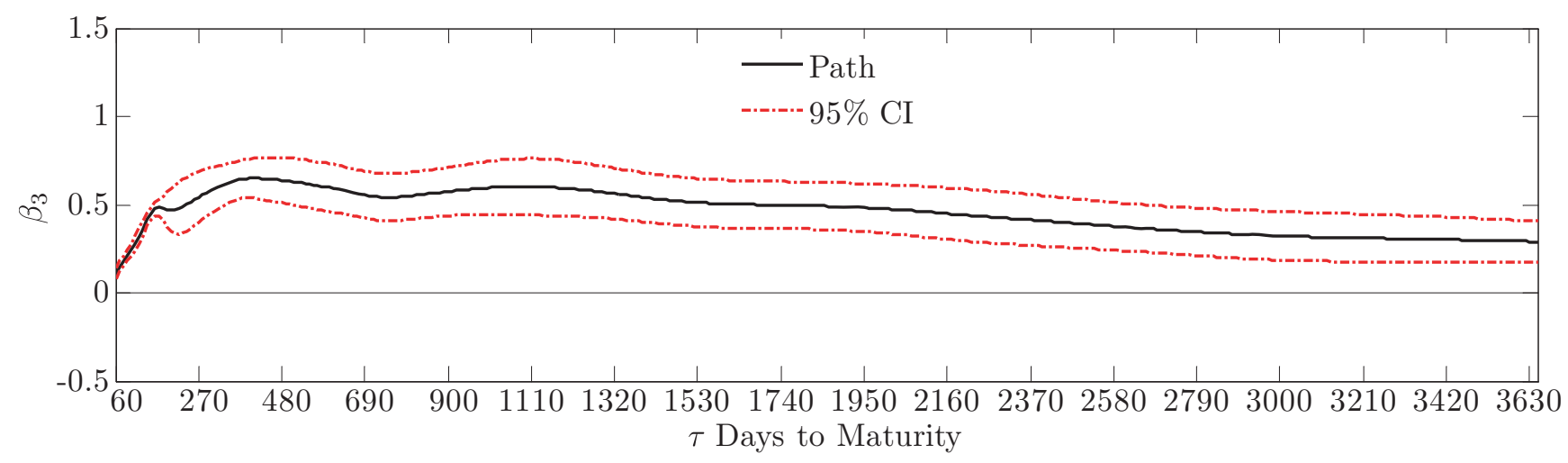

(c) Impact of path news on changes in yields with corrected asymptotic $95 \%$ confidence bounds

Figure 9: Impact of news from recursive regression analysis on yields of maturities $\tau=$ $60,70,80, \ldots, 3650$ (based on wide time window). 


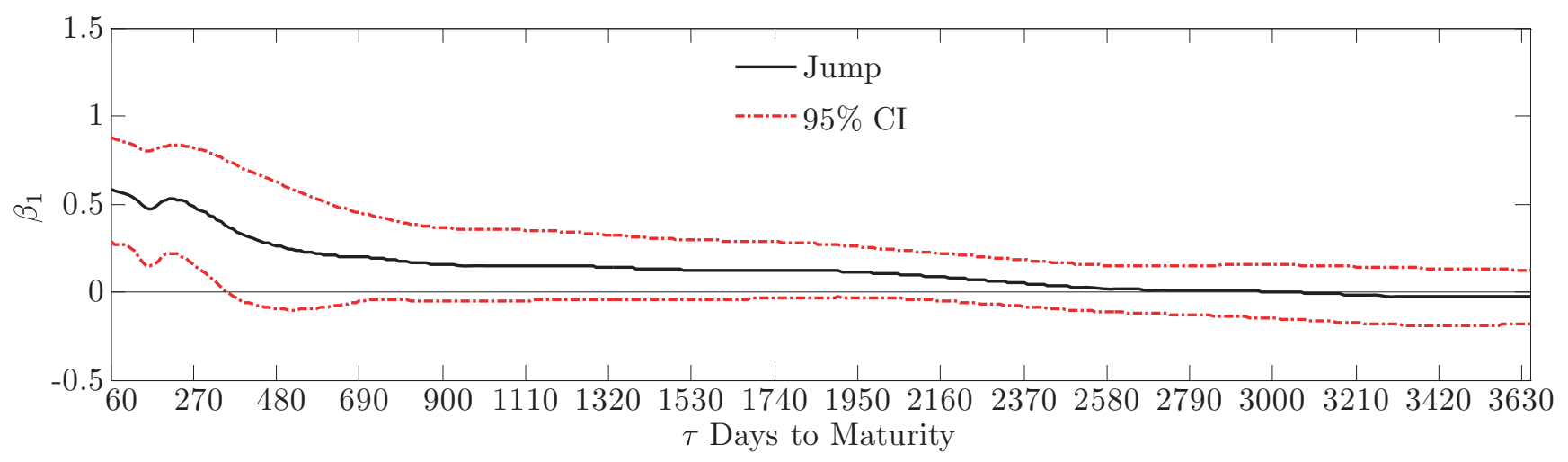

(a) Jump

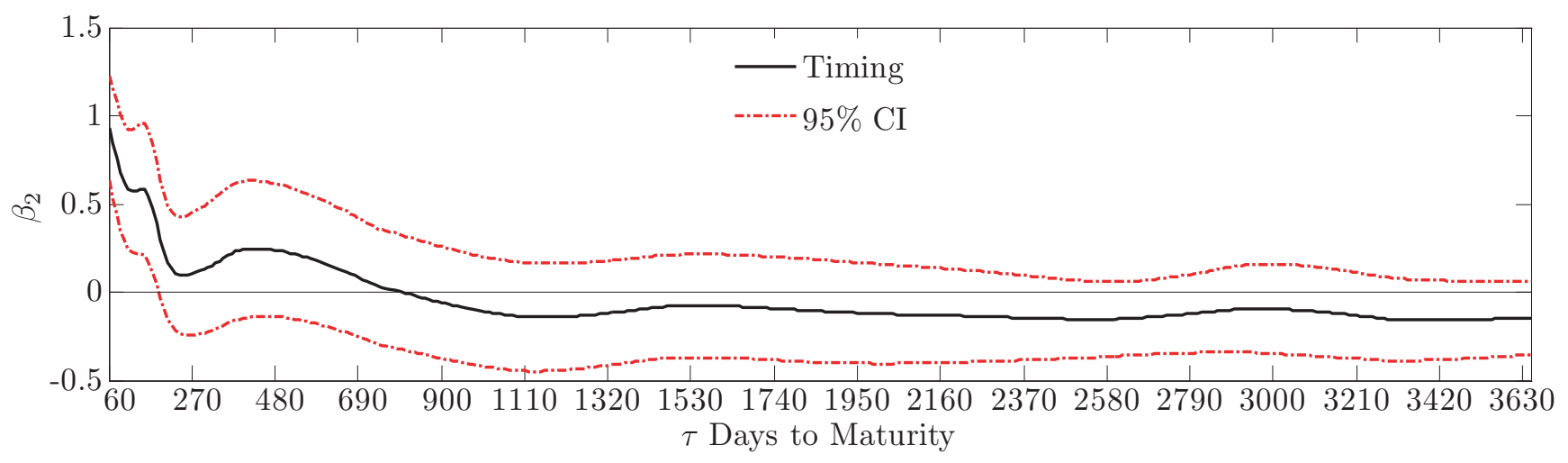

(b) Timing

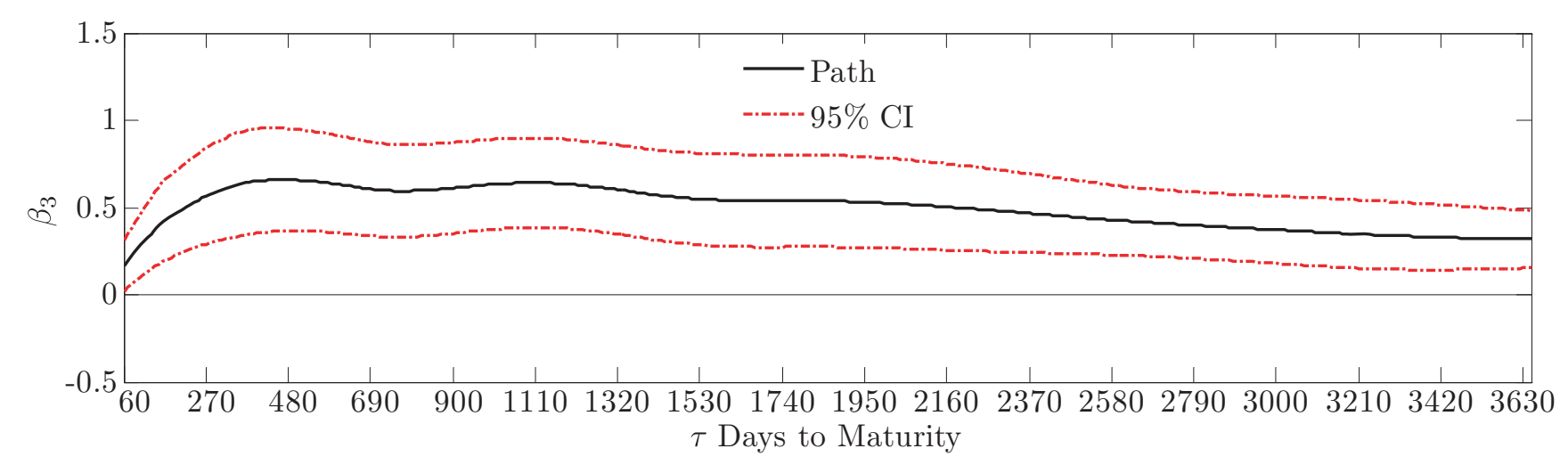

(c) Path

Figure 10: Impact of news from recursive regression analysis on yields of maturities $\tau=$ $60,70,80, \ldots, 3650$ (based on split-up decision and communication windows). 


\begin{tabular}{c|c|cc|c|ccc|c|ccc|c}
\hline \hline & & \multicolumn{3}{|c|}{$\begin{array}{c}\text { Principal } \\
\text { Components }\end{array}$} & \multicolumn{4}{c|}{$\begin{array}{c}\text { Recursive } \\
\text { Regressions }\end{array}$} & \multicolumn{4}{c}{ Partitioned } \\
& & \multicolumn{2}{|c|}{ Windows } \\
\hline Maturity & & Jump & Path & $R^{2}$ & Jump & Timing & Path & $R^{2}$ & Jump & Timing & Path & $R^{2}$ \\
\hline 2 year & US & - & - & - & $\mathbf{0 . 6 0 3}$ & $\mathbf{0 . 3 5 1}$ & $\mathbf{0 . 3 7 7}$ & 0.81 & - & - & - & - \\
180day & EUR & $\mathbf{0 . 4 8 1}$ & $\mathbf{0 . 4 7 3}$ & 0.80 & $\mathbf{0 . 8 0 5}$ & 0.181 & $\mathbf{0 . 4 8 0}$ & 0.97 & $\mathbf{0 . 4 9 5}$ & $\mathbf{0 . 3 8 8}$ & $\mathbf{0 . 4 5 0}$ & 0.74 \\
\hline 2 year & US & $\mathbf{0 . 4 8 2}$ & $\mathbf{0 . 4 1 1}$ & 0.94 & $\mathbf{0 . 6 4 3}$ & 0.096 & $\mathbf{0 . 9 5 7}$ & 0.69 & - & - & - & - \\
720day & EUR & $\mathbf{0 . 3 9 1}$ & $\mathbf{0 . 6 7 5}$ & 0.88 & $\mathbf{0 . 5 9 7}$ & 0.034 & $\mathbf{0 . 5 4 4}$ & 0.65 & 0.195 & 0.050 & $\mathbf{0 . 5 9 9}$ & 0.54 \\
\hline 5 year & US & $\mathbf{0 . 2 7 6}$ & $\mathbf{0 . 3 6 9}$ & 0.80 & $\mathbf{0 . 3 9 7}$ & -0.009 & $\mathbf{0 . 8 0 6}$ & 0.45 & - & - & - & - \\
1800day & EUR & $\mathbf{0 . 2 3 4}$ & $\mathbf{0 . 5 9 5}$ & 0.76 & $\mathbf{0 . 3 7 4}$ & -0.043 & $\mathbf{0 . 4 9 5}$ & 0.54 & 0.123 & -0.100 & $\mathbf{0 . 5 3 8}$ & 0.50 \\
\hline 10 year & US & $\mathbf{0 . 1 2 8}$ & $\mathbf{0 . 2 8 3}$ & 0.74 & 0.215 & 0.021 & $\mathbf{0 . 6 0 0}$ & 0.32 & - & - & - & - \\
3650day & EUR & $\mathbf{0 . 0 5 3}$ & $\mathbf{0 . 3 3 6}$ & 0.43 & 0.041 & -0.028 & $\mathbf{0 . 2 9 2}$ & 0.29 & -0.030 & -0.149 & $\mathbf{0 . 3 2 0}$ & 0.34 \\
\hline \hline
\end{tabular}

Table 1: Comparison of $\beta$ estimates from US to those of Euro (EUR) data. Bold entries denote significance at the 5\% level. Figures for the US entries are taken from Table 5 in Gürkaynak et al. (2005) and Table 3 in Gürkaynak (2005). 


\section{European Central Bank Working Paper Series}

For a complete list of Working Papers published by the ECB, please visit the ECB's website (http://www.ecb.int)

600 "A speed limit monetary policy rule for the euro area" by L. Stracca, April 2006.

601 "Excess burden and the cost of inefficiency in public services provision" by A. Afonso and V. Gaspar, April 2006.

602 "Job flow dynamics and firing restrictions: evidence from Europe" by J. Messina and G. Vallanti, April 2006.

603 "Estimating multi-country VAR models" by F. Canova and M. Ciccarelli, April 2006.

604 “A dynamic model of settlement” by T. Koeppl, C. Monnet and T. Temzelides, April 2006.

605 “(Un)Predictability and macroeconomic stability” by A. D’Agostino, D. Giannone and P. Surico, April 2006.

606 "Measuring the importance of the uniform nonsynchronization hypothesis" by D. A. Dias, C. Robalo Marques and J. M. C. Santos Silva, April 2006.

607 "Price setting behaviour in the Netherlands: results of a survey" by M. Hoeberichts and A. Stokman, April 2006.

608 "How does information affect the comovement between interest rates and exchange rates?" by M. Sánchez, April 2006.

609 "The elusive welfare economics of price stability as a monetary policy objective: why New Keynesian central bankers should validate core inflation” by W. H. Buiter, April 2006.

610 "Real-time model uncertainty in the United States: the Fed from 1996-2003" by R. J. Tetlow and B. Ironside, April 2006.

6 II "Monetary policy, determinacy, and learnability in the open economy" by J. Bullard and E. Schaling, April 2006.

612 "Optimal fiscal and monetary policy in a medium-scale macroeconomic model" by S. Schmitt-Grohé and M. Uribe, April 2006.

613 "Welfare-based monetary policy rules in an estimated DSGE model of the US economy" by M. Juillard, P. Karam, D. Laxton and P. Pesenti, April 2006.

614 "Expenditure switching vs. real exchange rate stabilization: competing objectives for exchange rate policy” by M. B. Devereux and C. Engel, April 2006.

$6 I 5$ “Quantitative goals for monetary policy” by A. Fatás, I. Mihov and A. K. Rose, April 2006.

616 "Global financial transmission of monetary policy shocks” by M. Ehrmann and M. Fratzscher, April 2006.

617 “New survey evidence on the pricing behaviour of Luxembourg firms” by P. Lünnemann and T. Y. Mathä, May 2006. 
618 "The patterns and determinants of price setting in the Belgian industry" by D. Cornille and M. Dossche, May 2006.

619 "Cyclical inflation divergence and different labor market institutions in the EMU" by A. Campolmi and E. Faia, May 2006.

620 "Does fiscal policy matter for the trade account? A panel cointegration study" by K. Funke and C. Nickel, May 2006.

621 "Assessing predetermined expectations in the standard sticky-price model: a Bayesian approach" by P. Welz, May 2006.

622 "Short-term forecasts of euro area real GDP growth: an assessment of real-time performance based on vintage data" by M. Diron, May 2006.

623 "Human capital, the structure of production, and growth" by A. Ciccone and E. Papaioannou, May 2006.

624 "Foreign reserves management subject to a policy objective" by J. Coche, M. Koivu, K. Nyholm and V. Poikonen, May 2006.

625 "Sectoral explanations of employment in Europe: the role of services" by A. D'Agostino, R. Serafini and M. Ward-Warmedinger, May 2006.

626 "Financial integration, international portfolio choice and the European Monetary Union" by R. A. De Santis and B. Gérard, May 2006.

627 "Euro area banking sector integration: using hierarchical cluster analysis techniques" by C. Kok Sørensen, J. M. Puigvert Gutiérrez, May 2006.

628 "Long-run money demand in the new EU Member States with exchange rate effects" by C. Dreger, H.-E. Reimers and B. Roffia, May 2006.

629 "A market microstructure analysis of foreign exchange intervention” by P. Vitale, May 2006.

630 “Implications of monetary union for catching-up member states” by M. Sánchez, May 2006.

631 "Which news moves the euro area bond market?" by M. Andersson, L. J. Hansen and S. Sebestyén, May 2006.

632 "Does information help recovering structural shocks from past observations?" by D. Giannone and L. Reichlin, May 2006.

633 "Nowcasting GDP and inflation: the real-time informational content of macroeconomic data releases" by D. Giannone, L. Reichlin and D. H. Small, May 2006.

634 "Expenditure reform in industrialised countries: a case study approach" by S. Hauptmeier, M. Heipertz and L. Schuknecht, May 2006.

635 "Identifying the role of labor markets for monetary policy in an estimated DSGE model" by K. Christoffel, K. Kuester and T. Linzert, June 2006.

636 "Exchange rate stabilization in developed and underdeveloped capital markets" by V. Chmelarova and G. Schnabl, June 2006. 
637 “Transparency, expectations, and forecasts” by A. Bauer, R. Eisenbeis, D. Waggoner and T. Zha, June 2006.

638 “Detecting and predicting forecast breakdowns” by R. Giacomini and B. Rossi, June 2006.

639 "Optimal monetary policy with uncertainty about financial frictions” by R. Moessner, June 2006.

640 “Employment stickiness in small manufacturing firms” by P. Vermeulen, June 2006.

64 I "A factor risk model with reference returns for the US dollar and Japanese yen bond markets" by C. Bernadell, J. Coche and K. Nyholm, June 2006.

642 “Financing constraints and firms' cash policy in the euro area” by R. Pál and A. Ferrando, June 2006.

643 "Inflation forecast-based-rules and indeterminacy: a puzzle and a resolution" by P. Levine, P. McAdam and J. Pearlman, June 2006.

644 “Adaptive learning, persistence, and optimal monetary policy” by V. Gaspar, F. Smets and D. Vestin, June 2006.

645 “Are internet prices sticky?” by P. Lünnemann and L. Wintr, June 2006.

646 "The Dutch block of the ESCB multi-country model” by E. Angelini, F. Boissay and M. Ciccarelli, June 2006.

647 "The economic effects of exogenous fiscal shocks in Spain: a SVAR approach" by F. de Castro Fernández and P. Hernández de Cos, June 2006.

648 "Firm-specific production factors in a DSGE model with Taylor price setting" by G. de Walque, F. Smets and R. Wouters, June 2006.

649 "Monetary and fiscal policy interactions in a New Keynesian model with capital accumulation and non-Ricardian consumers" by C. Leith and L. von Thadden, June 2006.

650 "A structural break in the effects of Japanese foreign exchange intervention on yen/dollar exchange rate volatility" by E. Hillebrand and G. Schnabl, June 2006.

651 "On the determinants of external imbalances and net international portfolio flows: a global perspective” by R. A. De Santis and M. Lührmann, July 2006.

652 "Consumer price adjustment under the microscope: Germany in a period of low inflation" by J. Hoffmann and J.-R. Kurz-Kim, July 2006.

653 "Acquisition versus greenfield: the impact of the mode of foreign bank entry on information and bank lending rates" by S. Claeys and C. Hainz, July 2006.

654 "The German block of the ESCB multi-country model” by I. Vetlov and T. Warmedinger, July 2006.

655 "Fiscal and monetary policy in the enlarged European Union” by S. Pogorelec, July 2006.

656 "Public debt and long-term interest rates: the case of Germany, Italy and the USA" by P. Paesani, R. Strauch and M. Kremer, July 2006. 
657 "The impact of ECB monetary policy decisions and communication on the yield curve" by C. Brand, D. Buncic and J. Turunen, July 2006. 
